\title{
Asymmetric thermal-relic dark matter: Sommerfeld-enhanced freeze-out, annihilation signals and unitarity bounds
}

\author{
Iason Baldes $^{a}$ and Kalliopi Petraki ${ }^{b, c}$ \\ ${ }^{a}$ DESY, Notkestraße 85, D-22607 Hamburg, Germany \\ ${ }^{b}$ Laboratoire de Physique Théorique et Hautes Energies (LPTHE), UMR 7589 CNRS \& \\ UPMC, 4 Place Jussieu, F-75252, Paris, France \\ ${ }^{c}$ Nikhef, Science Park 105, 1098 XG Amsterdam, The Netherlands \\ E-mail: iason.baldes@desy.de, kpetraki@lpthe.jussieu.fr
}

\begin{abstract}
Dark matter that possesses a particle-antiparticle asymmetry and has thermalised in the early universe, requires a larger annihilation cross-section compared to symmetric dark matter, in order to deplete the dark antiparticles and account for the observed dark matter density. The annihilation cross-section determines the residual symmetric component of dark matter, which may give rise to annihilation signals during CMB and inside haloes today. We consider dark matter with long-range interactions, in particular dark matter coupled to a light vector or scalar force mediator. We compute the couplings required to attain a final antiparticle-to-particle ratio after the thermal freeze-out of the annihilation processes in the early universe, and then estimate the late-time annihilation signals. We show that, due to the Sommerfeld enhancement, highly asymmetric dark matter with long-range interactions can have a significant annihilation rate, potentially larger than symmetric dark matter of the same mass with contact interactions. We discuss caveats in this estimation, relating to the formation of stable bound states. Finally, we consider the non-relativistic partial-wave unitarity bound on the inelastic cross-section, we discuss why it can be realised only by long-range interactions, and showcase the importance of higher partial waves in this regime of large inelasticity. We derive upper bounds on the mass of symmetric and asymmetric thermal-relic dark matter for $s$-wave and $p$-wave annihilation, and exhibit how these bounds strengthen as the dark asymmetry increases.
\end{abstract}




\section{Contents}

1 Introduction $\quad 1$

2 Thermal freeze-out in the presence of an asymmetry 3

2.1 The dark-sector temperature 3

2.2 Boltzmann equations 4

2.3 Dark matter mass and its maximum value 5

2.4 Final fractional asymmetry $r_{\infty} \quad 5$

3 Asymmetric freeze-out with Sommerfeld-enhanced cross-sections $\quad 8$

3.1 Vector mediator $\quad 8$

$\begin{array}{lll}3.2 & \text { Scalar mediator } & 13\end{array}$

4 Annihilation signals $\quad 15$

5 Unitarity limit $\quad 19$

$\begin{array}{llr}5.1 & \text { Long-range vs. contact-type interactions } & 19\end{array}$

$\begin{array}{lll}\text { 5.1.1 The velocity dependence of } \sigma_{\text {uni }} v_{\text {rel }} & 19\end{array}$

$\begin{array}{ll}\text { 5.1.2 Higher partial waves } & 20\end{array}$

5.2 Bounds on the mass of symmetric and asymmetric thermal-relic DM 22

6 Conclusion 23

\section{Introduction}

If dark matter (DM) transforms under a global U(1) symmetry that governs its low-energy interactions, it is possible that today there are unequal densities of dark particles and dark antiparticles. The dark particle-antiparticle asymmetry may have been related to the baryonic asymmetry of ordinary matter, via high-energy processes that occurred in the early universe, thereby providing a dynamical explanation for the similarity between the dark and the ordinary matter densities. Independently of such a connection, asymmetric DM can be a thermal relic of the primordial plasma while still having large couplings to lighter species, since its abundance cannot be depleted below the conserved excess of dark particles over antiparticles, via annihilations to these light species. Asymmetric DM thus provides a compelling cosmological scenario for large portions of the low-energy parameter space in a variety of beyond-the-Standard-Model theories, including models with new stable particles coupled to the Weak interactions of the Standard Model (WIMPs), as well as hidden-sector models [1].

In the asymmetric DM scenario, the efficiency of the annihilation processes in the early universe determines the relative abundance of dark particles and antiparticles today, i.e. the residual symmetric DM component. This, in turn, determines the DM annihilation signals at late times, that could be looked for by the ongoing indirect DM searches. The DM freeze-out in the presence of a particle-antiparticle asymmetry was first considered in [2,3], and more recently computed in greater detail and generality in [4,5]. Reference [4] showed that the residual dark antiparticle-to-particle ratio decreases exponentially with the DM annihilation 
cross-section. It then appears reasonable that sizeable annihilation signals may be expected only for annihilation cross-sections close to that for symmetric thermal-relic DM. This is indeed valid if DM annihilates via contact interactions, mediated by heavy particles $[4,6,7]$. This type of interactions were the focus of previous investigations [2-7].

In this paper, we consider asymmetric DM coupled to light force mediators. If a mediator is sufficiently light, then the interaction between DM particles manifests as long-range. More specifically, for an interaction that is described in the non-relativistic regime by a Yukawa potential, $V=-\alpha_{D} e^{-m_{\text {med }} r} / r$, long-range effects arise if the mediator mass is smaller than the Bohr momentum, $m_{\text {med }} \lesssim \alpha_{D} M_{\mathrm{DM}} / 2$, where $M_{\mathrm{DM}}$ is the DM mass. The long-range interaction distorts the wavefunction of the dark particle-antiparticle pairs, giving rise to the well-known Sommerfeld effect [8], which enhances the DM annihilation rate at low velocities $[9,10]$. In addition, long-range interactions imply the existence of bound states [11-18], whose formation is also a Sommerfeld-enhanced process [16]. The formation of unstable particle-antiparticle bound states, and their subsequent decay contributes to the overall DM annihilation rate. These non-perturbative phenomena, the Sommerfeld effect and the formation of bound states, on one hand suppress the couplings required to attain the observed DM density via thermal freeze-out in the early universe $[10,15]$; on the other hand, for a specified set of couplings, they enhance the late-time DM annihilation signals [11, 12, 18-24]. The present work aims to investigate the interplay between these two effects, in the context of asymmetric DM. Since the Sommerfeld enhancement depends on the coupling of DM to the light force mediator, and asymmetric DM requires stronger couplings than symmetric DM of the same mass, the implications for the phenomenology of asymmetric DM may be rather significant. ${ }^{1}$

We shall consider two minimal scenarios, in which DM is coupled to a massless or light vector boson, a dark photon, or to a light scalar mediator. We compute the couplings required to establish the observed DM abundance as a function of the dark asymmetry, and demonstrate the impact of the Sommerfeld effect. Using these computations, we estimate the strength of the radiative signals expected from the annihilation of the residual symmetric DM component inside haloes today. We find that highly asymmetric DM with long-range interactions can give rise to annihilation signals that are stronger than those of symmetric DM with contact interactions, up to several orders of magnitude. We discuss caveats to this estimate, related to the possible formation of stable bound states by asymmetric DM in the early universe.

Unitarity sets an upper limit on partial-wave inelastic cross-sections. This has been invoked to deduce the maximum mass for which thermalised DM can annihilate sufficiently in the early universe, to attain the observed density [26]. Asymmetric thermal-relic DM requires more efficient annihilation than symmetric DM; the upper mass bound implied by unitarity must, thus, tighten for larger values of the DM asymmetry. ${ }^{2}$ It has been pointed out that in the non-relativistic regime, the unitarity limit on the inelastic cross-section can be realised only via long-range interactions $[9,10,15]$. We recount the pertaining arguments, and further assert that, in the regime where the unitarity limit may be realised, partial waves

\footnotetext{
${ }^{1}$ A related computation of asymmetric freeze-out with Sommerfeld-enhanced cross-sections appeared recently in Ref. [25]. However, the scope and the extent of each study are very different.

${ }^{2}$ Asymmetric DM may be also produced non-thermally, in which case the computations of the present work, including the unitarity bounds, do not apply. The possibility of non-thermal asymmetric DM is encountered, for example, in the scenario of stable $Q$-balls produced in the fragmentation of an Affleck-Dine condensate [2729].
} 
beyond the lowest one, need to be considered. We then employ the freeze-out calculations with Sommerfeld-enhanced cross-sections carried out in this work, to compute the unitarity bounds on the mass and the asymmetry of thermal-relic DM, for the dominant partial waves that appear in known inelastic processes.

The rest of the paper is organised as follows. In section 2, we review the computation of the DM relic density in the presence of a conserved particle-antiparticle asymmetry. We follow closely the analysis of Ref. [4], and generalise it whenever necessary for our purposes. In section 3, we consider asymmetric DM coupled to light vector and scalar bosons, and compute the couplings required to establish the observed DM density, as a function of the DM asymmetry. In section 4, we estimate the expected indirect detection signals from the late-time annihilation of the residual symmetric DM component, and contemplate possible complications. In section 5, we discuss and compute the bounds implied by unitarity on symmetric and asymmetric thermal-relic DM. We conclude in section 6.

\section{Thermal freeze-out in the presence of an asymmetry}

\subsection{The dark-sector temperature}

The dark plasma - the bath of dark-sector relativistic particles into which DM annihilates - may be in general at a different temperature than photons. We will assume that at early times, the dark sector was in thermal equilibrium with the Standard Model (SM) plasma due to some unspecified high-energy interactions that decoupled at a high temperature $\tilde{T}$. Beyond this point, the SM and dark-sector temperatures, $T_{\mathrm{SM}}$ and $T_{D}$, evolve differently. The SM-sector, dark-sector and total entropy densities are $s_{\mathrm{SM}}=\left(2 \pi^{2} / 45\right) g_{\mathrm{SM}} T_{\mathrm{SM}}^{3}, s_{D}=$ $\left(2 \pi^{2} / 45\right) g_{D} T_{D}^{3}$ and $s=s_{\mathrm{SM}}+s_{D}$ respectively, where $g_{\mathrm{SM}}$ and $g_{D}$ are the SM and dark-sector relativistic degrees of freedom, which depend on the temperatures. Assuming conservation of co-moving entropy in each sector separately below the common temperature $\tilde{T}$, the darkto-ordinary temperature ratio is

$$
\tau \equiv \frac{T_{D}}{T_{\mathrm{SM}}}=\left(\frac{g_{\mathrm{SM}}}{g_{D}}\right)^{1 / 3}\left(\frac{\tilde{g}_{D}}{\tilde{g}_{\mathrm{SM}}}\right)^{1 / 3},
$$

where $\tilde{g}_{\mathrm{SM}}$ and $\tilde{g}_{D}$ refer to the temperature $\tilde{T}$. For our purposes, it will be convenient to express the total entropy and energy densities in terms of $T_{D}$,

$$
s=\left(2 \pi^{2} / 45\right) h_{\mathrm{eff}}\left(T_{D}\right) T_{D}^{3}, \quad \rho=\left(\pi^{2} / 30\right) g_{\mathrm{eff}}\left(T_{D}\right) T_{D}^{4},
$$

where ${ }^{3}$

$$
\begin{aligned}
h_{\mathrm{eff}} & \equiv g_{\mathrm{SM}} / \tau^{3}+g_{D}=g_{D}\left(1+\tilde{g}_{\mathrm{SM}} / \tilde{g}_{D}\right), \\
g_{\mathrm{eff}} & \equiv g_{\mathrm{SM}} / \tau^{4}+g_{D}=g_{D}\left[1+\left(g_{D} / g_{\mathrm{SM}}\right)^{1 / 3}\left(\tilde{g}_{\mathrm{SM}} / \tilde{g}_{D}\right)^{4 / 3}\right] .
\end{aligned}
$$

The Hubble parameter in the radiation dominated epoch is $H=\sqrt{4 \pi^{3} g_{\mathrm{eff}} / 45} T_{D}^{2} / M_{\mathrm{Pl}}$.

We use the values of $g_{\mathrm{SM}}$ available with the MicrOMEGAs package [30], and assume that $\tilde{g}_{\mathrm{SM}}$ includes all the SM degrees of freedom. We shall take $\tilde{g}_{D}=5$ or 6 , to account for the degrees of freedom of DM consisting of Dirac Fermions, plus a real scalar or vector force

\footnotetext{
${ }^{3}$ Here, we do not distinguish between the entropy and the energy degrees of freedom for the SM and the dark sector. For the temperature range of interest, there is no difference.
} 
mediator respectively. At $T_{D}<\tilde{T}$, we take $g_{D}=\tilde{g}_{D}$ for $T_{D} \gtrsim M_{\mathrm{DM}} / 3$ and $g_{D}=\tilde{g}_{D}-4$ for $T_{D}<M_{\mathrm{DM}} / 3{ }^{4}$

\subsection{Boltzmann equations}

We shall use $x_{D} \equiv M_{\mathrm{DM}} / T_{D}$ as the time variable, and parametrise the thermally averaged annihilation cross-section times relative velocity as

$$
\left\langle\sigma_{\mathrm{ann}} v_{\mathrm{rel}}\right\rangle \equiv \sigma_{*} \times F\left(x_{D}\right) .
$$

For fully perturbative $s$ - and $p$-wave annihilation $F\left(x_{D}\right)=1$ and $F\left(x_{D}\right)=\left\langle v_{\text {rel }}^{2}\right\rangle=6 / x_{D}$ respectively; however, for an inelastic process well within the Sommerfeld-enhanced regime and the Coulomb approximation, $F\left(x_{D}\right) \propto x_{D}^{1 / 2}$, independently of the partial wave it may be dominated by $[15,16,33]$. More details will be specified in section 3 .

The DM particle and antiparticle number-to-entropy ratios, $Y^{ \pm} \equiv n^{ \pm} / s$, evolve according to

$$
\frac{d Y^{ \pm}}{d x_{D}}=-\frac{g_{*}^{1 / 2} \lambda F\left(x_{D}\right)}{x_{D}^{2}}\left[Y^{+}\left(x_{D}\right) Y^{-}\left(x_{D}\right)-Y_{\mathrm{eq}}^{\mathrm{sym}}\left(x_{D}\right)^{2}\right]
$$

where

$$
\begin{aligned}
g_{*}^{1 / 2} & \equiv \frac{h_{\mathrm{eff}}}{g_{\mathrm{eff}}^{1 / 2}}\left(1+\frac{T_{D}}{4 g_{\mathrm{eff}}} \frac{d g_{\mathrm{eff}}}{d T_{D}}\right), \\
\lambda & \equiv \sqrt{\frac{\pi}{45}} \sigma_{*} M_{\mathrm{DM}} M_{\mathrm{Pl}}, \\
Y_{\mathrm{eq}}^{\mathrm{sym}}\left(x_{D}\right) & \simeq \frac{90}{(2 \pi)^{7 / 2}} \frac{g_{X}}{h_{\mathrm{eff}}} x_{D}^{3 / 2} e^{-x_{D}} .
\end{aligned}
$$

In the above, $Y_{\mathrm{eq}}^{\mathrm{sym}}$ is the equilibrium value of $Y^{ \pm}$in the absence of an asymmetry, with $g_{X}$ being the DM degrees of freedom. In the presence of an asymmetry, the equilibrium values of $Y^{ \pm}$are

$$
Y_{\mathrm{eq}}^{ \pm}\left(x_{D}\right)=Y_{\mathrm{eq}}^{\mathrm{sym}}\left(x_{D}\right) e^{ \pm \xi_{D}},
$$

where $\xi_{D} \equiv \mu / T_{D}$, with $\mu$ being the chemical potential, which evolves with $T_{D}$ in order to account for the conserved dark particle-antiparticle asymmetry, as we shall now see [cf. eq. (2.7)].

We define two asymmetry parameters, the fractional asymmetry $r$ and the dark particleminus-antiparticle-number-to-entropy ratio $\eta_{D}$,

$$
\begin{aligned}
r\left(x_{D}\right) & \equiv Y^{-}\left(x_{D}\right) / Y^{+}\left(x_{D}\right), \\
\eta_{D} & \equiv Y^{+}-Y^{-} .
\end{aligned}
$$

\footnotetext{
${ }^{4}$ Under these assumptions, a massless or very light mediator $\left(m_{\text {med }} \lesssim \mathrm{eV}\right)$ would contribute to the relativistic energy density during $\mathrm{CMB}$ by $\delta N_{\mathrm{eff}} \approx 0.47$, which is compatible with current constraints within about $2.5 \sigma$ [31]. This tension is alleviated if the mediator is somewhat massive and either decays sufficiently early into SM particles via a portal operator (see e.g. Ref. [24] for relevant considerations on a particular model), or becomes non-relativistic after its density has redshifted to a negligible amount, but before CMB. Alternatively, there may be additional degrees of freedom coupled to the SM sector at $\tilde{T}$ whose later decoupling from the SM thermal bath would suppress the dark-to-ordinary temperature ratio $\tau$ and lower $\delta N_{\text {eff }}$. Moreover, it is possible that the dark sector was at a lower temperature in early times due to initial conditions set by inflation (see e.g. [32]). Of course, a lower dark-sector temperature would also affect the DM freeze-out and decrease the estimated couplings that can produce the observed DM density; the estimated annihilation cross-section at the time of freeze-out would have to be lower by approximately the same factor as the dark-sector temperature.
} 
Equations (2.5) and (2.6) can be inverted to give $Y^{+}=\eta_{D} /(1-r)$ and $Y^{-}=\eta_{D} r /(1-r)$. In an isentropically expanding universe, $\eta_{D}$ is conserved. While DM is in chemical equilibrium with dark radiation, the fractional asymmetry is $r_{\mathrm{eq}} \equiv Y_{\mathrm{eq}}^{-} / Y_{\mathrm{eq}}^{+}=\exp \left(-2 \xi_{D}\right)$, where the equilibrium chemical potential is determined from eqs. (2.4d) and (2.6) to be

$$
\xi_{D}\left(x_{D}\right)=\ln \left[\sqrt{1+\left(\frac{\eta_{D}}{2 Y_{\mathrm{eq}}^{\mathrm{sym}}\left(x_{D}\right)}\right)^{2}}+\frac{\eta_{D}}{2 Y_{\mathrm{eq}}^{\mathrm{sym}}\left(x_{D}\right)}\right] .
$$

Ultimately, we are interested in computing the final fractional asymmetry,

$$
r_{\infty} \equiv \lim _{x_{D} \rightarrow \infty} r\left(x_{D}\right),
$$

which determines the DM annihilation signals today, and the value of the predicted the DM mass.

\subsection{Dark matter mass and its maximum value}

The ratio of DM to ordinary matter relic energy densities is $\Omega_{\mathrm{DM}} / \Omega_{\mathrm{B}}=\left(Y_{\infty}^{+}+Y_{\infty}^{-}\right) M_{\mathrm{DM}} /\left(\eta_{B} m_{p}\right)$, where $\eta_{B}$ is the baryon-number-to-entropy ratio of the universe, and $m_{p}$ is the proton mass. Using eqs. (2.5) and (2.6), and setting $\epsilon \equiv \eta_{D} / \eta_{B}$, we obtain

$$
M_{\mathrm{DM}}=\frac{m_{p}}{\epsilon} \frac{\Omega_{\mathrm{DM}}}{\Omega_{\mathrm{B}}}\left(\frac{1-r_{\infty}}{1+r_{\infty}}\right) .
$$

For non-zero $\epsilon$, eq. (2.9) implies a maximum DM mass

$$
M_{\mathrm{DM}}<M_{\max }(\epsilon) \equiv \frac{m_{p}}{\epsilon} \frac{\Omega_{\mathrm{DM}}}{\Omega_{\mathrm{B}}} \simeq 5 \mathrm{GeV} / \epsilon,
$$

attained in the limit $r_{\infty} \rightarrow 0 .{ }^{5}$ This would, however, require an infinitely large cross-section. Partial-wave unitarity sets an upper limit on the inelastic cross-section, and thus implies $r_{\infty}>0$, which in turn strengthens the upper bound on the mass of (asymmetric) thermalrelic DM to $M_{\mathrm{DM}}<M_{\mathrm{uni}}<M_{\max }$. In section 5 , we show how $M_{\text {uni }}$ varies with the DM asymmetry.

\subsection{Final fractional asymmetry $r_{\infty}$}

From eq. (2.4), we find that $r$ is governed by the equation [4]

$$
\frac{d r}{d x_{D}}=-\frac{\eta_{D} \lambda g_{*}^{1 / 2} F\left(x_{D}\right)}{x_{D}^{2}}\left[r-r_{\mathrm{eq}}\left(\frac{1-r}{1-r_{\mathrm{eq}}}\right)^{2}\right] .
$$

Soon after freeze-out, the second term in eq. (2.11) becomes unimportant, and the evolution of $r$ is determined by the first term. The final fractional asymmetry can thus be approximated by

$$
r_{\infty} \simeq r_{\mathrm{eq}}^{\mathrm{FO}} \exp \left[-\eta_{D} \lambda \Phi\left(\alpha_{D}\right)\right]
$$

where

$$
\Phi\left(\alpha_{D}\right) \equiv \int_{x_{D}^{\mathrm{FO}}}^{\infty} d x_{D} g_{*}^{1 / 2} F\left(x_{D}\right) / x_{D}^{2}
$$

\footnotetext{
${ }^{5}$ Equivalently, eq. (2.9) implies a maximum asymmetry $\epsilon<\epsilon_{\max }\left(M_{\mathrm{DM}}\right) \simeq 5 \mathrm{GeV} / M_{\mathrm{DM}}$.
} 
Here, we have chosen to emphasise the possible dependence of $\Phi$ on the couplings of the theory (denoted by $\alpha_{D}$ ) that arises in Sommerfeld-enhanced cross-sections (to be specified in section 3). This is important for the determination of $r_{\infty}$, as discussed below. In the cases of interest, the function $F\left(x_{D}\right)$ either decreases with $x_{D}$ or grows at most as $x_{D}^{1 / 2}$, therefore the integral (2.13) is dominated by the contribution at $x_{D} \approx x_{D}^{\mathrm{FO}}$, and can be approximated as

$$
\Phi\left(\alpha_{D}\right) \simeq \frac{\sqrt{g_{*}^{\mathrm{FO}}} F\left(x_{D}^{\mathrm{FO}}\right)}{c_{\Phi} x_{D}^{\mathrm{FO}}},
$$

where $c_{\Phi} \sim \mathcal{O}(1)$ is a numerical factor that will not appear in our final result below. Freeze-out - the time when the densities of the DM particles and antiparticles depart from their equilibrium values - occurs when the terms contributing to the logarithmic derivatives of the dark particle and antiparticle densities, $d \ln Y^{ \pm} / d x_{D}$, become small, i.e. at $\lambda \sqrt{g_{*}^{\mathrm{FO}}} F\left(x_{D}^{\mathrm{FO}}\right) Y_{\mathrm{eq}}^{\mathrm{sym}}\left(x_{D}^{\mathrm{FO}}\right) /\left(x_{D}^{\mathrm{FO}}\right)^{2} \sim 1 / c_{x}$ [cf. eq. (2.4)], where $c_{x} \sim \mathcal{O}(1)$. This yields the standard algebraic equation for $x_{D}^{\mathrm{FO}}$, which in our formalism reads

$$
x_{D}^{\mathrm{FO}}+(1 / 2) \ln x_{D}^{\mathrm{FO}}-\ln F\left(x_{D}^{\mathrm{FO}}\right) \simeq \ln \left(c_{x} 0.15 \lambda g_{X} / \sqrt{g_{*}^{\mathrm{FO}}}\right) .
$$

Note that $x_{D}^{\mathrm{FO}} \approx 20-30$ is insensitive to the presence of an asymmetry [4]. Using eq. (2.13a), $Y_{\mathrm{eq}}^{\mathrm{sym}}$ can be re-expressed as $Y_{\mathrm{eq}}^{\mathrm{sym}}\left(x_{D}^{\mathrm{FO}}\right) \approx x_{D}^{\mathrm{FO}} /(c \lambda \Phi)$, where $c \sim c_{\Phi} c_{x}$. From eq. (2.7), we may now estimate the fractional asymmetry at freeze-out,

$$
r_{\mathrm{eq}}^{\mathrm{FO}} \approx\left[\sqrt{1+\left(c \eta_{D} \lambda \Phi\right)^{2} /\left(2 x_{D}^{\mathrm{FO}}\right)^{2}}+\left(c \eta_{D} \lambda \Phi\right) /\left(2 x_{D}^{\mathrm{FO}}\right)\right]^{-2} .
$$

For an interaction that scales as $F\left(x_{D}\right) \propto x_{D}^{-n}, c_{x} \approx c_{\Phi}=n+1$ (see e.g. Ref. [34]); consequently $c \approx(n+1)^{2}$.

From eqs. (2.12) and (2.15), we see that $r_{\infty}$ depends on the the combination of parameters $\eta_{D} \lambda \Phi$. A direct comparison with symmetric DM can be established by recasting $\eta_{D}$ in terms of $r_{\infty}$ using eq. (2.9), and recalling that in the symmetric DM limit, $\Omega_{\mathrm{DM}} / \Omega_{\mathrm{B}}=\left(2 Y_{\infty}^{\mathrm{sym}} M_{\mathrm{DM}}\right) /\left(\eta_{B} m_{p}\right)$ where the relic number-to-entropy ratio is

$$
Y_{\infty}^{\mathrm{sym}} \simeq\left[\left(1+c / x_{D}^{\mathrm{FO}}\right) \lambda_{\mathrm{sym}} \Phi_{\mathrm{sym}}\right]^{-1}
$$

as can be deduced from eq. (2.4). Then, we find

$$
\eta_{D} \lambda \Phi=\frac{2}{1+c / x_{D}^{\mathrm{FO}}}\left(\frac{1-r_{\infty}}{1+r_{\infty}}\right)\left(\frac{\sigma_{*}}{\sigma_{*, \mathrm{sym}}}\right)\left(\frac{\Phi}{\Phi_{\mathrm{sym}}}\right)
$$

where the subscript "sym" refers to symmetric DM of the same mass.

Collecting the above, we obtain ${ }^{6}$

$$
r_{\infty} \simeq \frac{\exp \left(-\eta_{D} \lambda \Phi\right)}{\left[\sqrt{1+\left(\frac{c \eta_{D} \lambda \Phi}{2 x_{D}^{\mathrm{FO}}}\right)^{2}}+\left(\frac{c \eta_{D} \lambda \Phi}{2 x_{D}^{\mathrm{FO}}}\right)\right]^{2}} .
$$

For a small asymmetry $\eta_{D}$, we expand $\ln \left(r_{\infty}\right) \approx-\left(1+c / x_{D}^{\mathrm{FO}}\right) \eta_{D} \lambda \Phi+\mathcal{O}\left[\left(c / x_{D}^{\mathrm{FO}}\right)^{3} \eta_{D}^{3} \lambda^{3} \Phi^{3}\right]$. Keeping only the lowest order term is a good approximation for $\left(c / x_{D}^{\mathrm{FO}}\right) \eta_{D} \lambda \Phi \lesssim 1$; because

\footnotetext{
${ }^{6}$ This expression is more general than the expressions provided in Ref. [4].
} 
$c / x_{D}^{\mathrm{FO}} \ll 1$, this range extends to very small $r_{\infty}$. Then, using eq. (2.17), we arrive at the result of Ref. [4],

$$
r_{\infty} \simeq \exp \left[-2\left(\frac{1-r_{\infty}}{1+r_{\infty}}\right)\left(\frac{\sigma_{*}}{\sigma_{*, \mathrm{sym}}}\right)\left(\frac{\Phi}{\Phi_{\mathrm{sym}}}\right)\right] .
$$

Note that using the approximation $(2.13 \mathrm{a}),\left(\sigma_{*} \Phi\right) /\left(\sigma_{* \text {,sym }} \Phi_{\mathrm{sym}}\right) \simeq \sigma_{\mathrm{ann}}^{\mathrm{FO}} / \sigma_{\mathrm{ann}, \mathrm{sym}}^{\mathrm{FO}}$, provided that $F\left(x_{D}\right)$ scales with $x_{D}$ in the same way, around the time of freeze-out, for the couplings corresponding to the symmetric and asymmetric cases.

As is evident from eqs. (2.18), $r_{\infty}$ depends exponentially on the annihilation crosssection at freeze-out. Therefore, a cross-section only somewhat larger than that required for symmetric thermal-relic DM, suffices to diminish the antiparticle density considerably [4]. For annihilation via fully perturbative processes, the dependence of the cross-section on the couplings of the theory and on the DM velocity (or temperature) can be factorised inside $\sigma_{*}$ and $F\left(x_{D}\right)$ respectively; $\Phi$ depends only on $x_{D}^{\mathrm{FO}}$, which is insensitive to $\sigma_{*}$ (i.e. the couplings of the theory), thus $\Phi \simeq \Phi_{\text {sym }}$. This case was investigated in detail in Ref. [4]. However, for Sommerfeld-enhanced cross-sections, this factorisation is not in general possible; $\Phi$ depends on the couplings of the theory explicitly (rather than via $x_{D}^{\mathrm{FO}}$ only), and can differ significantly from $\Phi_{\text {sym }}$. This enhances the sensitivity of $r_{\infty}$ to the strength of the interactions, and implies that a small $r_{\infty}$ can be attained for more modest couplings. For an annihilation cross-section that scales around the time of freeze-out as $\sigma_{\mathrm{ann}}^{\mathrm{FO}} \propto \alpha_{D}^{p}$, we find from eq. (2.18a),

$$
\alpha_{D} / \alpha_{D}^{\mathrm{sym}} \simeq\left[\left(\frac{1+r_{\infty}}{1-r_{\infty}}\right) \frac{\ln \left(1 / r_{\infty}\right)}{2}\right]^{1 / p} .
$$

We investigate the effect of the Sommerfeld enhancement for specific interactions in the next section. 


\section{Asymmetric freeze-out with Sommerfeld-enhanced cross-sections}

We will consider two minimal cases, in which DM consists of Dirac Fermions and couples either to a light vector or scalar boson. In both cases, the interaction between dark particles and antiparticles is described in the non-relativistic regime by a static Yukawa potential, $V_{Y}(\mathbf{r})=-\alpha_{D} e^{-m_{\operatorname{med}} r} / r$. We will perform all computations in the Coulomb limit, $m_{\text {med }} \rightarrow$ 0 , which is a satisfactory approximation if the average momentum transfer between the interacting particles is larger than the mediator mass, $v_{\text {rel }} M_{\mathrm{DM}} / 2 \gtrsim m_{\text {med }}$ [18]. The Coulomb approximation is suitable during the DM chemical decoupling in the early universe, essentially in the entire range where non-perturbative effects arise $\left(m_{\text {med }} \lesssim \alpha_{D} M_{\mathrm{DM}} / 2\right)$ [24].

\subsection{Vector mediator}

We consider the interaction Lagrangian

$$
\mathcal{L}=\bar{X}\left(i \not D-M_{\mathrm{DM}}\right) X-\frac{1}{4} F_{D \mu \nu} F_{D}^{\mu \nu},
$$

where $X$ denotes the DM particle, with covariant derivative $D^{\mu}=\partial^{\mu}+i g_{d} V_{D}^{\mu}$, and $F_{D}^{\mu \nu}=$ $\partial^{\mu} V_{D}^{\nu}-\partial^{\nu} V_{D}^{\mu}$, with $V_{D}^{\mu}$ being the dark photon field and $\alpha_{D} \equiv g_{d}^{2} /(4 \pi)$ being the dark finestructure constant. If $X$ carries a particle-antiparticle asymmetry, another field is required to balance the implied $U(1)_{D}$ charge asymmetry in $X$; we return to the implications of this in section 4 .

Two processes contribute significantly to the depletion of DM in the early universe [15]: the direct annihilation into two dark photons, and the radiative formation of positronium-like bound states followed by their decay,

$$
\begin{aligned}
X+\bar{X} \rightarrow & 2 V_{D}, \\
X+\bar{X} \rightarrow & \mathcal{B}_{s}(\bar{X} X)+V_{D}(\omega), \\
& \mathcal{B}_{\uparrow \downarrow}(\bar{X} X) \rightarrow 2 V_{D}, \\
& \mathcal{B}_{\uparrow \uparrow}(\bar{X} X) \rightarrow 3 V_{D},
\end{aligned}
$$

where the subscript $s=\uparrow \downarrow, \uparrow \uparrow$ denotes the spin-singlet and spin-triplet bound states, which form with $25 \%$ and $75 \%$ probability, respectively. The dark photon emitted during boundstate formation (BSF) carries away energy $\omega=\Delta+E_{k}$, where $\Delta=M_{\mathrm{DM}} \alpha_{D}^{2} / 4$ is the binding energy and $E_{k}=M_{\mathrm{DM}} v_{\mathrm{rel}}^{2} / 4$ is the kinetic energy of the two incoming particles in the centerof-momentum frame. The Feynman diagrams for the processes (3.2) and (3.3) are shown in fig. 1.

The (spin-averaged) cross-sections for annihilation and radiative capture to the ground state can be expressed as $[15,16,18]$

$$
\begin{aligned}
& \sigma_{\mathrm{ann}} v_{\mathrm{rel}}=\sigma_{0} S_{\mathrm{ann}}^{(0)}, \\
& \sigma_{\mathrm{BSF}} v_{\mathrm{rel}}=\sigma_{0} S_{\mathrm{BSF}},
\end{aligned}
$$

where

$$
\sigma_{0} \equiv \pi \alpha_{D}^{2} / M_{\mathrm{DM}}^{2}
$$

is the perturbative value of the annihilation cross-section times relative velocity. ${ }^{7}$ In the Coulomb limit, $S_{\mathrm{ann}}^{(0)}$ and $S_{\mathrm{BSF}}$ depend only on the ratio $\zeta \equiv \alpha_{D} / v_{\text {rel }}$, and can be computed

\footnotetext{
${ }^{7}$ The superscript "(0)" in $S_{\text {ann }}^{(0)}$ denotes that the annihilation into two vector bosons is an $s$-wave process, at leading order in $\alpha_{D}$ and $v_{\text {rel }}$. This is important for the unitarity bounds on $M_{\mathrm{DM}}$ that we discuss in section 5 .
} 


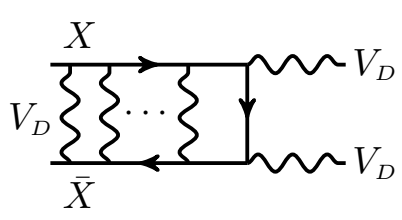

(a)
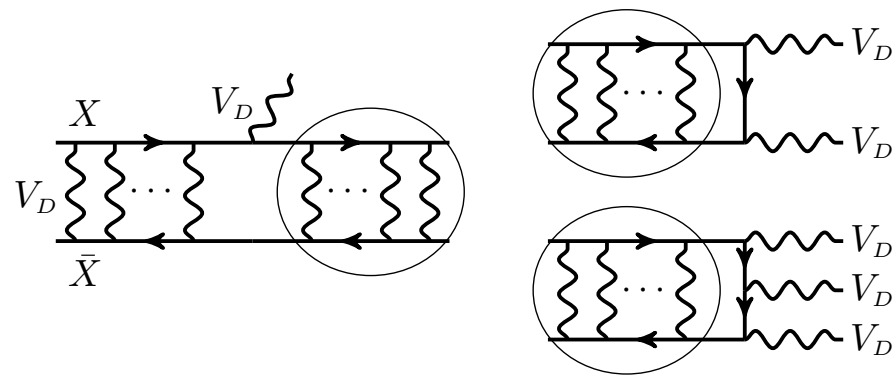

(b)

Figure 1. Dark matter coupled to a light or massless dark photon $V_{D}$ can annihilate either (a) directly into radiation, or (b) in two steps, via the radiative formation of particle-antiparticle bound states, and their subsequent decay into two or three dark photons, for the spin-singlet (para) and spintriplet (ortho) configurations respectively. Both the direct annihilation and the formation of bound states are enhanced by the Sommerfeld effect (initial-state ladder); in the Coulomb regime, boundstate formation is faster than annihilation whenever the Sommerfeld effect is important $\left(v_{\text {rel }} \lesssim \alpha_{D}\right)$.

analytically $[15,16,18]$,

$$
\begin{aligned}
S_{\mathrm{ann}}^{(0)}(\zeta) & =\frac{2 \pi \zeta}{1-e^{-2 \pi \zeta}}, \\
S_{\mathrm{BSF}}(\zeta) & =\frac{2 \pi \zeta}{1-e^{-2 \pi \zeta}} \frac{\zeta^{4}}{\left(1+\zeta^{2}\right)^{2}} \frac{2^{9}}{3} e^{-4 \zeta \operatorname{arccot}(\zeta)} .
\end{aligned}
$$

In this parametrisation, it is easily seen that in the regime where the Sommerfeld effect is important, $v_{\text {rel }} \lesssim \alpha_{D}$, both the annihilation and BSF cross-sections exhibit the same velocity dependence, $\sigma v_{\text {rel }} \propto 1 / v_{\text {rel }}$, with BSF being the dominant inelastic process, $\sigma_{\mathrm{BSF}} / \sigma_{\text {ann }} \simeq$ $3.13[15] .{ }^{8}$ On the other hand, for $v_{\text {rel }}>\alpha_{D}$, BSF is very suppressed and subdominant to annihilation. The bound-state decay rates are $\Gamma_{\mathrm{dec}, \uparrow \downarrow}=\alpha^{5} M_{\mathrm{DM}} / 2$ and $\Gamma_{\mathrm{dec}, \uparrow \uparrow}=c_{\alpha_{D}} \Gamma_{\mathrm{dec}, \uparrow \downarrow}$, where $c_{\alpha_{D}} \equiv 4\left(\pi^{2}-9\right) \alpha_{D} /(9 \pi)$.

The evolution of the DM density in the early universe is governed by a set of coupled equations which tracks the densities of the unbound DM particles and anti-particles, as well as the densities of the bound states. These equations capture the effect of direct annihilation and pair creation, as well as the interplay between bound-state formation, ionisation and decay processes that determines the efficiency of BSF in depleting DM. Because the velocity dependence of $\sigma_{\mathrm{ann}}$ and $\sigma_{\mathrm{BSF}}$ arises via the parameter $\zeta=\alpha_{D} / v_{\mathrm{rel}}=\left(\Delta / E_{k}\right)^{1 / 2}$, the thermally-averaged cross-sections depend on

$$
z_{D} \equiv \Delta / T_{D}=\left(\alpha_{D}^{2} / 4\right) x_{D}
$$

We shall use $z_{D}$ are the time variable, and denote with $Y_{\uparrow \downarrow}$ and $Y_{\uparrow \uparrow}$ the number-density-toentropy ratios for the spin-singlet and triplet states. Adapting the Boltzmann equations from

\footnotetext{
${ }^{8}$ In the same regime, the capture into $n=2, \ell=1$ bound states is also somewhat faster than annihilation [18]. However, it is subdominant with respect to the capture to the ground state $(n=1, \ell=0)$, and has a smaller decay rate, which renders it less efficient in depleting DM in the early universe. We shall ignore it in our analysis.
} 
Ref. [15] to accommodate for a non-zero particle-antiparticle asymmetry, we obtain ${ }^{9}$

$$
\begin{aligned}
\frac{d r}{d z_{D}}= & -\frac{\eta_{D} \lambda_{1} g_{*}^{1 / 2} \bar{S}_{\mathrm{ann}}^{(0)}\left(z_{D}\right)}{z_{D}^{2}}\left[r-r_{\mathrm{eq}}\left(\frac{1-r}{1-r_{\mathrm{eq}}}\right)^{2}\right] \\
& -\frac{\eta_{D} \lambda_{1} g_{*}^{1 / 2} \bar{S}_{\mathrm{BSF}}\left(z_{D}\right)}{z_{D}^{2}} r+\frac{\lambda_{2} g_{*}^{1 / 2} z_{D} f_{\mathrm{ion}}\left(z_{D}\right)\left(Y_{\uparrow \downarrow}+Y_{\uparrow \uparrow}\right)(1-r)^{2}}{\eta_{D} h_{\mathrm{eff}}} \\
\frac{d Y_{\uparrow \downarrow}}{d z_{D}}= & \frac{\eta_{D}^{2} \lambda_{1} g_{*}^{1 / 2} \bar{S}_{\mathrm{BSF}}\left(z_{D}\right)}{4 z_{D}^{2}} \frac{r}{(1-r)^{2}}-\frac{\lambda_{2} g_{*}^{1 / 2} z_{D} f_{\mathrm{ion}}\left(z_{D}\right) Y_{\uparrow \downarrow}}{h_{\mathrm{eff}}}-\frac{\lambda_{2} g_{*}^{1 / 2} z_{D}\left(Y_{\uparrow \downarrow}-Y_{\uparrow \downarrow}^{\mathrm{eq}}\right)}{h_{\mathrm{eff}}},(3.8 \mathrm{~b}) \\
\frac{d Y_{\uparrow \uparrow}}{d z_{D}}= & \frac{3 \eta_{D}^{2} \lambda_{1} g_{*}^{1 / 2} \bar{S}_{\mathrm{BSF}}\left(z_{D}\right)}{4 z_{D}^{2}} \frac{r}{(1-r)^{2}}-\frac{\lambda_{2} g_{*}^{1 / 2} z_{D} f_{\mathrm{ion}}\left(z_{D}\right) Y_{\uparrow \uparrow}}{h_{\mathrm{eff}}}-\frac{\lambda_{2} g_{*}^{1 / 2} c_{\alpha_{D}} z_{D}\left(Y_{\uparrow \uparrow}-Y_{\uparrow \uparrow}^{\mathrm{eq}}\right)}{h_{\mathrm{eff}}},
\end{aligned}
$$

where $\lambda_{1} \equiv \sqrt{\pi / 45} \sigma_{0} \Delta M_{\mathrm{Pl}}, \lambda_{2} \equiv \sqrt{45 /\left(4 \pi^{3}\right)}\left(\alpha_{D}^{5} M_{\mathrm{DM}} / 2\right)\left(M_{\mathrm{Pl}} / \Delta^{2}\right)$ and

$$
\begin{aligned}
\bar{S}_{\mathrm{ann}}^{(0)}\left(z_{D}\right) & \equiv \frac{2}{\sqrt{\pi}} \int_{0}^{\infty} d u S_{\mathrm{ann}}^{(0)}\left(\sqrt{z_{D} / u}\right) \sqrt{u} \exp (-u), \\
\bar{S}_{\mathrm{BSF}}\left(z_{D}\right) & \equiv \frac{2}{\sqrt{\pi}} \int_{0}^{\infty} d u S_{\mathrm{ann}}^{(0)}\left(\sqrt{z_{D} / u}\right) \frac{\sqrt{u} \exp (-u)}{1-\exp \left(-z_{D}-u\right)}, \\
f_{\mathrm{ion}}\left(z_{D}\right) & \equiv \frac{\Gamma_{\mathrm{ion}}\left(z_{D}\right)}{\Gamma_{\mathrm{dec}, \uparrow \downarrow}}=\frac{1}{8 \pi} \int_{0}^{\infty} \frac{d \zeta}{\zeta^{4}} \frac{S_{\mathrm{BSF}}(\zeta)}{\exp \left[z_{D}\left(1+1 / \zeta^{2}\right)\right]-1} .
\end{aligned}
$$

Here, $\bar{S}_{\text {ann }}^{(0)}$ is the thermal average of $S_{\text {ann }}^{(0)}$, while $\bar{S}_{\mathrm{BSF}}$ is the thermally averaged $S_{\mathrm{BSF}}$ times the Bose enhancement due to the final-state dark photon emitted during BSF [cf. eq. (3.3)]. $\Gamma_{\text {ion }}\left(z_{D}\right)$ is the bound-state ionisation rate, averaged over the dark photon thermal bath, and depends on $S_{\mathrm{BSF}}$ because the amplitudes for inverse processes are related. For more details, see Ref. [15]. We present the results of our computation in fig. 2.

As described in Ref. [15], the formation of bound states depletes efficiently the DM density only after the bound-state decay becomes faster than ionisation. The decay rate of the spin-singlet bound state becomes larger than ionization at $f_{\text {ion }}\left(z_{D}\right) \lesssim 1$, or $z_{D} \gtrsim 0.28$. For the spin-triplet state, this occurs at a later time, when $f_{\text {ion }}\left(z_{D}\right) \lesssim c_{\alpha_{D}}$. We may thus adopt the following approximation for the evolution of $r$,

$$
\frac{d r}{d z_{D}}=-\frac{\eta_{D} \lambda_{1} g_{*}^{1 / 2} S_{\mathrm{eff}}\left(z_{D}\right)}{z_{D}^{2}}\left[r-r_{\mathrm{eq}}\left(\frac{1-r}{1-r_{\mathrm{eq}}}\right)^{2}\right]
$$

where $S_{\text {eff }}$ is defined as [15]

$$
S_{\mathrm{eff}}\left(z_{D}\right) \equiv \begin{cases}\bar{S}_{\mathrm{ann}}\left(z_{D}\right), & z_{D} \lesssim 0.28 \\ \bar{S}_{\mathrm{ann}}\left(z_{D}\right)+\bar{S}_{\mathrm{BSF}}\left(z_{D}\right) / 4, & 0.28 \lesssim z_{D} \text { and } c_{\alpha_{D}} \lesssim f_{\mathrm{ion}}\left(z_{D}\right) \\ \bar{S}_{\mathrm{ann}}\left(z_{D}\right)+\bar{S}_{\mathrm{BSF}}\left(z_{D}\right), & f_{\mathrm{ion}}\left(z_{D}\right) \lesssim c_{\alpha_{D}}\end{cases}
$$

\footnotetext{
${ }^{9}$ Equations (3.8) include the bound-state inverse decays, which were omitted in Ref. [15]. Their effect is negligible.
} 


\section{Vector mediator}
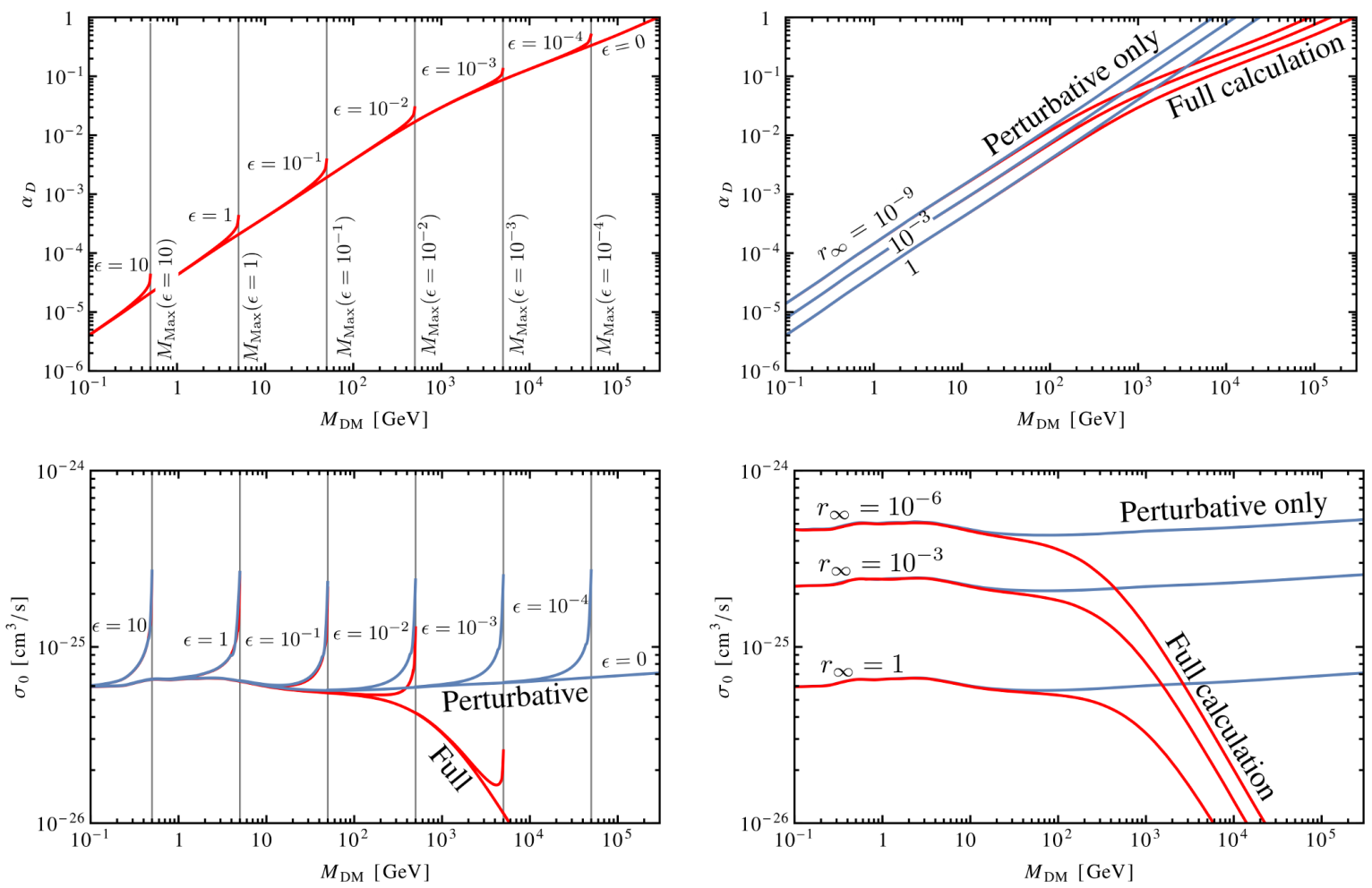

Figure 2. Top: The dark fine-structure constant required to establish the observed DM density via thermal freeze-out, $\alpha_{D}$ vs $M_{\mathrm{DM}}$, for fixed values of the dark particle-minus-antiparticle-numberto-entropy ratio $\eta_{D}=\epsilon \eta_{B}$ (left), and for fixed values of the final antiparticle-to-particle ratio $r_{\infty} \equiv$ $\left(n^{+} / n^{-}\right)_{t \rightarrow \infty}($ right $)$. The red lines include both the Sommerfeld enhancement of the direct DM annihilation into radiation, and the formation and decay of particle-antiparticle bound states. The blue lines ignore all non-perturbative effects.

Bottom: The perturbative annihilation cross-section times relative velocity, $\sigma_{0}=\pi \alpha_{D}^{2} / M_{\mathrm{DM}}^{2}$ vs $M_{\mathrm{DM}}$, for fixed $\epsilon=\eta_{D} / \eta_{B}$ (left), and $r_{\infty}$ (right). $\sigma_{0}$ is evaluated using $\alpha_{D}$ determined as described above. For $M_{\mathrm{DM}} \ll M_{\max }(\epsilon)$ or $r_{\infty} \approx 1, \alpha_{D}$ and $\sigma_{0}$ closely track the symmetric DM curve $(\epsilon=0)$. For $r_{\infty} \ll 1$ to be attained and $M_{\mathrm{DM}} \simeq M_{\max }$ to be realised, a stronger coupling is required. The stronger coupling implies that the Sommerfeld effect — which reduces the expected coupling in comparison to perturbative annihilation - is more pronounced for smaller $r_{\infty}$, and extends to lower $M_{\mathrm{DM}}$ values.

This approximation ${ }^{10}$ produces results that are in very good agreement with those obtained from the full treatment of eqs. (3.8). Moreover, eq. (3.10) can be mapped to the discussion of section 2, and in particular eq. (2.11), by identifying

$$
\begin{aligned}
\sigma_{*} & \rightarrow \sigma_{0}, \\
F\left(x_{D}\right) & \rightarrow S_{\mathrm{eff}}\left(z_{D}\right),
\end{aligned}
$$

\footnotetext{
${ }^{10}$ A similar prescription for $S_{\text {eff }}$, with a smoother transition between regimes, has been offered in Ref. [17]. Adapted to the present model, it reads

$$
S_{\mathrm{eff}}\left(z_{D}\right) \equiv \bar{S}_{\mathrm{ann}}\left(z_{D}\right)+\frac{1}{1+f_{\mathrm{ion}}\left(z_{D}\right)} \frac{\bar{S}_{\mathrm{BSF}}\left(z_{D}\right)}{4}+\frac{c_{\alpha_{D}}}{c_{\alpha_{D}}+f_{\mathrm{ion}}\left(z_{D}\right)} \frac{3 \bar{S}_{\mathrm{BSF}}\left(z_{D}\right)}{4} .
$$
}




\section{Vector mediator}
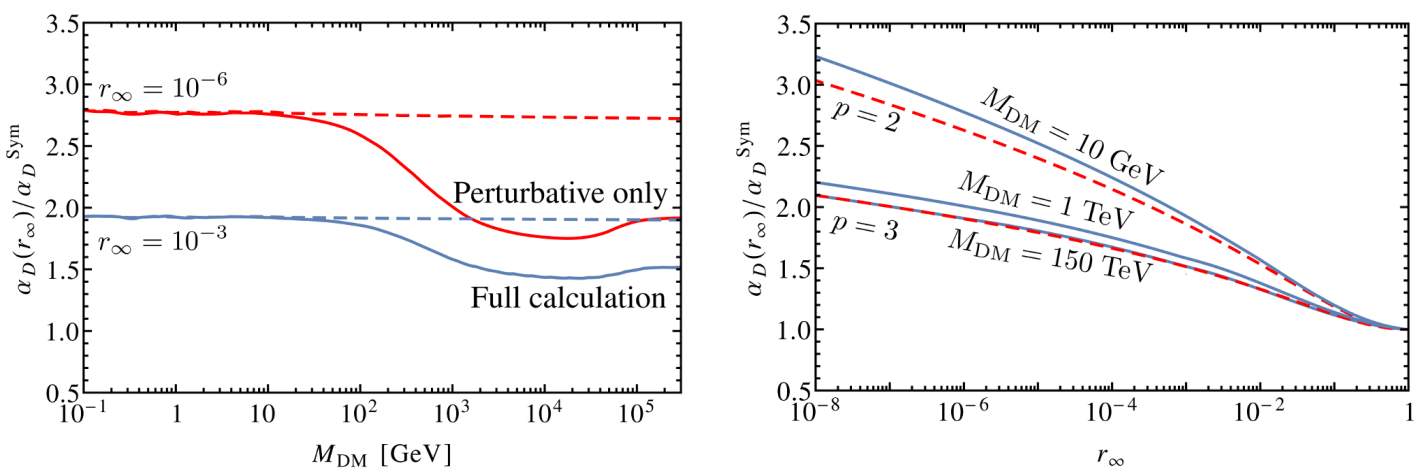

Figure 3. The ratio of the coupling required to establish a fractional asymmetry $r_{\infty}$ to the coupling required for symmetric DM of the same mass. The larger the asymmetry (i.e. the smaller $r_{\infty}$ ), the larger $\alpha_{D}\left(r_{\infty}\right) / \alpha_{D}^{\mathrm{sym}}$.

Left: $\alpha_{D}\left(r_{\infty}\right) / \alpha_{D}^{\mathrm{sym}}$ vs $M_{\mathrm{DM}}$, for $r_{\infty}=10^{-3}$ (blue) and $r_{\infty}=10^{-6}$ (red). Annihilation via Sommerfeld enhancement processes (solid lines) implies a lower $\alpha_{D}\left(r_{\infty}\right) / \alpha_{D}^{\mathrm{sym}}$ in comparison to perturbative annihilation (dashed lines). For small (large) $M_{\mathrm{DM}}$, freeze-out occurs well within the perturbative (Sommerfeld enhanced) regime, where the inelastic cross-sections scale as $\sigma_{\text {inel }}^{\text {FO }} \propto \alpha_{D}^{p}$, with $p=2$ $(p=3)$. Within these regimes, $\alpha_{D}\left(r_{\infty}\right) / \alpha_{D}^{\text {sym }}$ is largely independent of the DM mass for a fixed $r_{\infty}$, as anticipated by the analytical approximation of eq. (2.19). (The mild dependence of $\alpha_{D}\left(r_{\infty}\right) / \alpha_{D}^{\mathrm{sym}}$ on $M_{\mathrm{DM}}$ at large $M_{\mathrm{DM}}$, is due to the intricacy of the effect of bound states on the DM relic density. See footnote 12 for discussion.)

Right: $\alpha_{D}\left(r_{\infty}\right) / \alpha_{D}^{\text {sym }}$ vs $r_{\infty}$, for $M_{\mathrm{DM}}=10 \mathrm{GeV}, 1 \mathrm{TeV}$ and $150 \mathrm{TeV}$ (blue solid lines, from top to bottom). The red dashed lines denote the analytical approximation of eqs. (2.18) and (2.19), assuming that the inelastic cross-sections around the time of freeze-out scale as $\sigma_{\text {inel }}^{\text {FO }} \propto \alpha_{D}^{p}$, with $p=2$ for fully perturbative annihilation (upper line), and $p=3$ for Sommerfeld-enhanced annihilation (lower line). For intermediate masses (e.g. $M_{\mathrm{DM}} \sim 1 \mathrm{TeV}$ ), the scaling of $\sigma_{\text {inel }}^{\mathrm{FO}}$ with $\alpha_{D}$, and consequently the scaling of $\alpha_{D}\left(r_{\infty}\right) / \alpha_{D}^{\mathrm{sym}}$ with $r_{\infty}$, fall in between these two cases.

where $z_{D}$ and $x_{D}$ are related via eq. (3.7).

We discern two regimes, the perturbative and the Sommerfeld-enhanced. The Sommerfeld enhancement is important for $\sqrt{z_{D}} \simeq\left\langle\alpha / v_{\text {rel }}\right\rangle \gtrsim 1 .{ }^{11}$ For small $M_{\mathrm{DM}}$, freeze-out occurs in the perturbative regime, $z_{D}^{\mathrm{FO}} \ll 1$; then $F\left(x_{D}^{\mathrm{FO}}\right) \simeq 1, \Phi \simeq \Phi_{\mathrm{sym}} \simeq \sqrt{g_{*}^{\mathrm{FO}}} / x_{D}^{\mathrm{FO}}$ and $\sigma_{*} \Phi \propto \alpha_{D}^{2}$. On the other hand, for larger $M_{\mathrm{DM}}$, freeze-out happens close to or within the Sommerfeldenhanced regime. When well within the Sommerfeld-enhanced regime, $F\left(x_{D}\right) \sim z_{D}^{1 / 2}, \Phi \sim$ $\alpha_{D} \sqrt{g_{*}^{\mathrm{FO}}} / x_{D}^{1 / 2}$ and $\sigma_{*} \Phi \propto \alpha_{D}^{3}$. Then, from eq. (2.19), we may estimate the coupling $\alpha_{D}\left(r_{\infty}\right)$ required to establish a fractional asymmetry $r_{\infty}$, by setting $p=2$ for small $M_{\mathrm{DM}}$, and $p=3$ for large $M_{\mathrm{DM}}$. It is anticipated that in the small and large $M_{\mathrm{DM}}$ limits, the ratio $\alpha_{D} / \alpha_{D}^{\mathrm{sym}}$ scales solely with $r_{\infty}$, and is insensitive to $M_{\mathrm{DM}}$ for fixed $r_{\infty}$. The numerical solution for $\alpha_{D} / \alpha_{D}^{\text {sym }}$ is presented in fig. 3, and indeed exhibits the two asymptotic behaviours described here. ${ }^{12}$ Note that because $\alpha_{D}$ increases with decreasing $r_{\infty}$, the mass scale of the transition

\footnotetext{
${ }^{11}$ While $x_{D}^{\mathrm{FO}} \approx 25-30$ is insensitive to $M_{\mathrm{DM}}, z_{D}^{\mathrm{FO}}=\left(\alpha_{D}^{2} / 4\right) x_{D}^{\mathrm{FO}}$ increases with $\alpha_{D}$ and consequently $M_{\mathrm{DM}}$.

12 The mild sensitivity of $\alpha_{D} / \alpha_{D}^{\mathrm{sym}}$ on $M_{\mathrm{DM}}$ in the Sommerfeld enhanced regime (large $M_{\mathrm{DM}}$ ) arises from the numerical coefficient in $F$ and consequently $\sigma_{*} \Phi$, which depends on whether and which BSF channels contribute to the depletion of the DM density, as described by $S_{\text {eff }}$ defined in eq. (3.10a). Since for the same $M_{\mathrm{DM}}, \alpha_{D}$ increases with decreasing $r_{\infty}$, the DM depletion via BSF may be more efficient for $r_{\infty} \ll 1$ than for $r_{\infty}=1$. For example, for $M_{\mathrm{DM}} \sim 10 \mathrm{TeV}$, only the formation of spin-singlet bound states contributes
} 
between the perturbative and the Sommerfeld-enhanced regimes depends on $r_{\infty}$.

\subsection{Scalar mediator}

The interaction Lagrangian is

$$
\mathcal{L}=\bar{X}\left(i \not \partial-M_{\mathrm{DM}}\right) X+\frac{1}{2} \partial_{\mu} \varphi \partial^{\mu} \varphi-\frac{1}{2} m_{\varphi}^{2} \varphi^{2}-g_{d} \varphi \bar{X} X,
$$

with $\varphi$ being the dark scalar force mediator with mass $m_{\varphi}$, and $\alpha_{D} \equiv g_{d}^{2} /(4 \pi)$. As long as $m_{\varphi} \lesssim \alpha_{D} M_{\mathrm{DM}} / 2$, the $X-\bar{X}$ interaction manifests as long range.

For the determination of the DM relic density, which occurs largely in the Coulomb limit [24], only the DM direct annihilation into two scalars, $X+\bar{X} \rightarrow 2 \varphi$, needs be considered, since it is significantly faster than BSF [22]. The annihilation cross-section times relative velocity is

$$
\sigma_{\mathrm{ann}} v_{\mathrm{rel}}=\sigma_{1} v_{\mathrm{rel}}^{2} S_{\mathrm{ann}}^{(1)},
$$

where $^{13}$

$$
\begin{aligned}
\sigma_{1} & =\frac{3 \pi \alpha_{D}^{2}}{8 M_{\mathrm{DM}}^{2}}, \\
S_{\mathrm{ann}}^{(1)}(\zeta) & =\frac{2 \pi \zeta}{1-e^{-2 \pi \zeta}}\left(1+\zeta^{2}\right) .
\end{aligned}
$$

As before, $\zeta \equiv \alpha_{D} / v_{\text {rel }}$. At $v_{\text {rel }} \lesssim \alpha_{D}$, the cross-section exhibits the familiar velocity scaling of Sommerfeld enhanced processes, $\sigma_{\text {ann }} v_{\text {rel }} \propto 1 / v_{\text {rel }}$. In this regime, the $v_{\text {rel }}^{2}$ suppression of the perturbative cross-section morphs into an $\alpha_{D}^{2}$ suppression,,${ }^{14}$ with the entire cross-section scaling as $\sigma_{\text {ann }} v_{\text {rel }} \propto \alpha_{D}^{5}$.

The evolution of the fractional asymmetry $r$ is determined from eq. (2.11), if we identify

$$
\begin{aligned}
\sigma_{*} & \rightarrow \sigma_{1}, \\
F\left(x_{D}\right) & \rightarrow\left\langle v_{\text {rel }}^{2} S_{\text {ann }}^{(1)}(\zeta)\right\rangle=\alpha_{D}^{2}\left\langle S_{\text {ann }}^{(1)}(\zeta) / \zeta^{2}\right\rangle,
\end{aligned}
$$

where the thermal average of eq. $(3.14 \mathrm{~b})$ is

$$
F\left(x_{D}\right)=\frac{8}{\sqrt{\pi} x_{D}} \int_{0}^{\infty} d u S_{\text {ann }}^{(1)}\left(\sqrt{z_{D} / u}\right) u^{3 / 2} \exp (-u) .
$$

We compute the coupling $\alpha_{D}$ required to obtain the observed DM relic density, as a function of the asymmetry, and present the results in fig. 4 .

As in section 3.1 for a vector mediator, we discern the perturbative and the Sommerfeldenhanced regimes. In the perturbative regime, $F\left(x_{D}^{\mathrm{FO}}\right) \simeq\left\langle v_{\mathrm{rel}}^{2}\right\rangle_{\mathrm{FO}}=6 / x_{D}^{\mathrm{FO}}, \Phi \simeq \Phi_{\mathrm{sym}} \simeq$ $3 \sqrt{g_{*}^{\mathrm{FO}}} /\left(x_{D}^{\mathrm{FO}}\right)^{2}$, and $\sigma_{*} \Phi \propto \alpha_{D}^{2}$. Well within the Sommerfeld-enhanced regime, $F\left(x_{D}^{\mathrm{FO}}\right) \simeq$ $2 \alpha_{D}^{3} \sqrt{\pi x_{D}^{\mathrm{FO}}}, \Phi \simeq \alpha_{D}^{3} \sqrt{\pi g_{*}^{\mathrm{FO}} / x_{D}^{\mathrm{FO}}}$ and $\sigma_{*} \Phi \propto \alpha_{D}^{5}$. Then, from eq. (2.19), we estimate the ratio $\alpha_{D} / \alpha_{D}^{\text {sym }}$ required to establish a fractional asymmetry $r_{\infty}$, by setting $p=2$ and $p=5$ for small and large $M_{\mathrm{DM}}$ respectively. We present the numerical solution for $\alpha_{D} / \alpha_{D}^{\mathrm{sym}}$ in fig. 5 , and compare it with the analytical approximation.

to the depletion of symmetric DM, while both spin-singlet and spin-triplet bound states deplete DM with $r_{\infty} \sim 10^{-6}$. On the other hand, for $M_{\mathrm{DM}} \sim 100 \mathrm{TeV}$, both BSF channels contribute to $S_{\text {eff }}$ for any $r_{\infty}$.

${ }^{13}$ The superscript "(1)" in $S_{\text {ann }}^{(1)}$ denotes that the annihilation of two fermions into two scalar bosons is a $p$-wave process. This is important for the unitarity bounds discussed in section 5 .

${ }^{14}$ The physical significance of this transformation is the following. Due to the long-range nature of the interaction, the two incoming particles are not momentum eigenstates. While their relative momentum has expectation value $\mu v_{\text {rel }}$, its dispersion is $\sim \mu \alpha_{D}$, where $\mu$ is the reduced mass. 


\section{Scalar mediator}
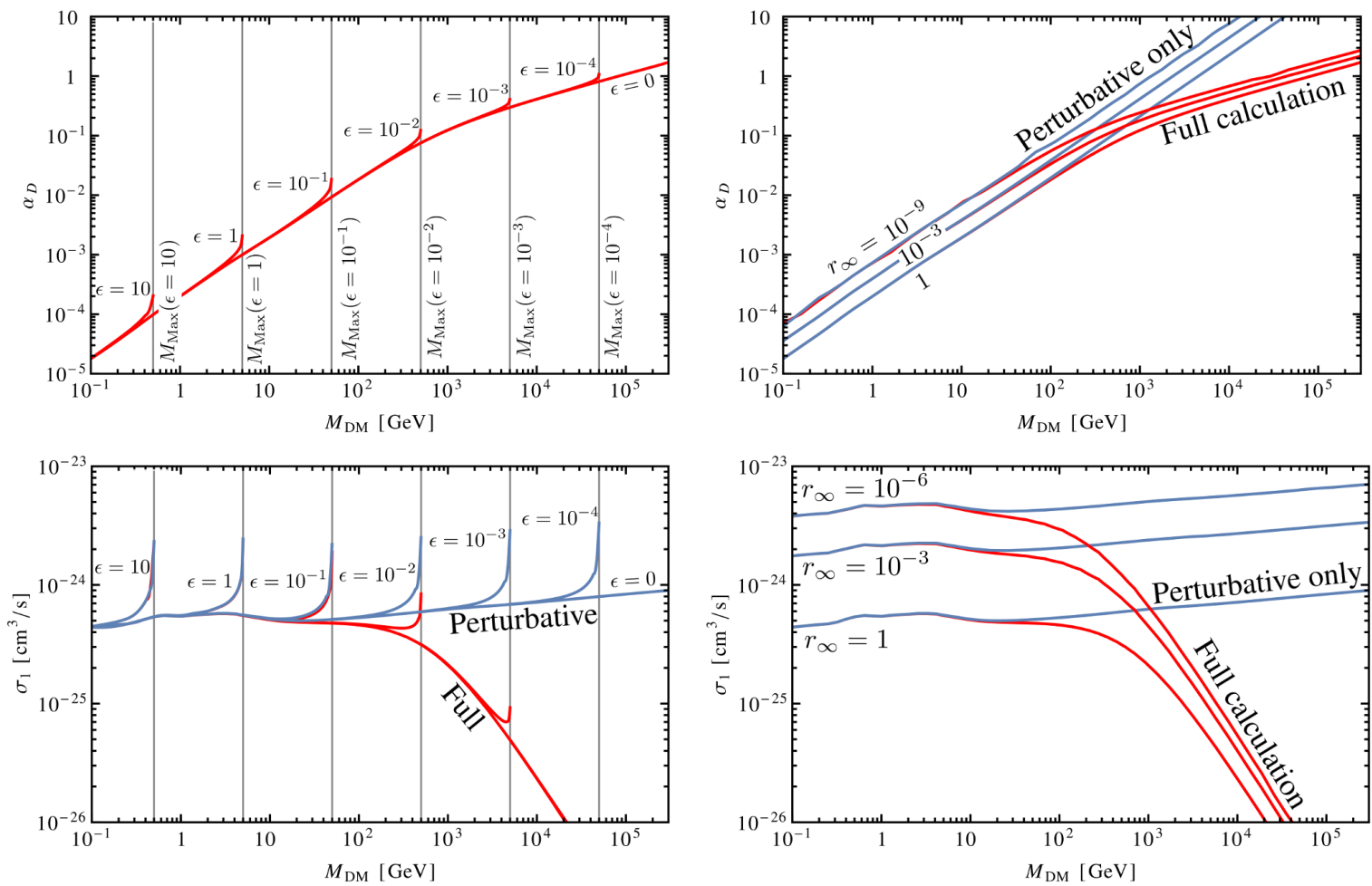

Figure 4. The equivalent of fig. 2 , for DM coupled to a light scalar. Top: $\alpha_{D}$ vs $M_{\mathrm{DM}}$. Bottom: $\sigma_{1} \equiv 3 \pi \alpha_{D}^{2} /\left(8 M_{\mathrm{DM}}^{2}\right)$ vs $M_{\mathrm{DM}}$.
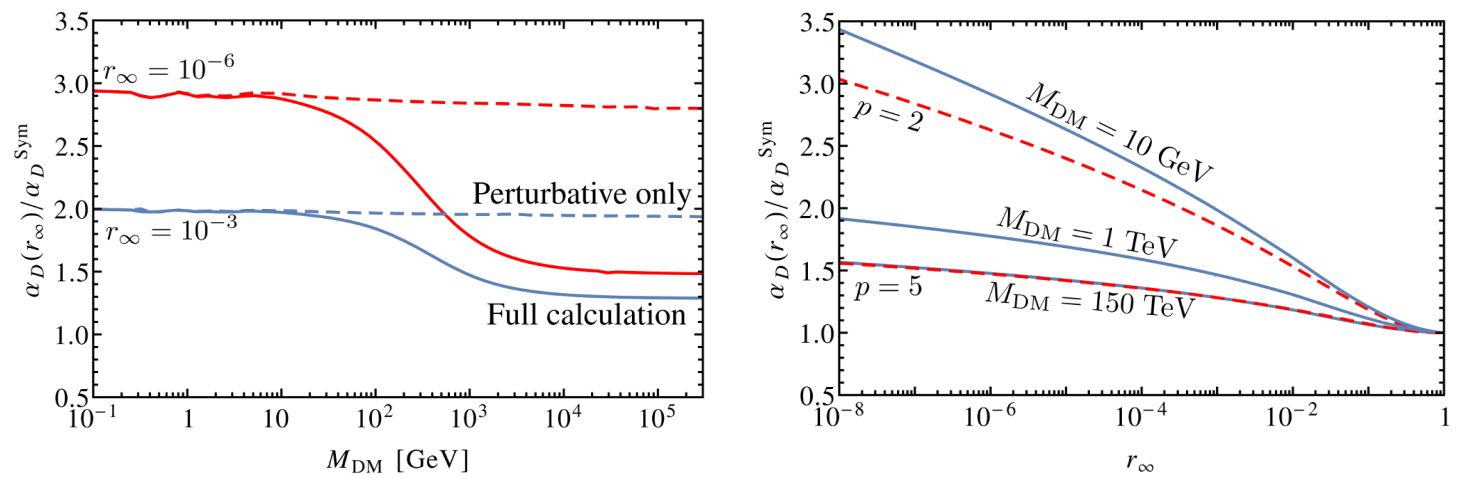

Figure 5. The equivalent of fig. 3, for DM coupled to a light scalar mediator. The annihilation cross-section around the time of freeze out scales as $\sigma_{\text {ann }}^{\mathrm{FO}} \propto \alpha_{D}^{p}$, with $p=2$ for small $M_{\mathrm{DM}}$ and $p=5$ for large $M_{\mathrm{DM}}$. 


\section{Annihilation signals}

The residual symmetric DM component may give rise to DM annihilation that could have observable implications [4]. Provided that the DM annihilation products include or cascade down to SM states, the DM annihilation at late cosmic times may be constrained by CMB observations [35], while the DM annihilation inside haloes today may yield detectable signals $[6,7]$. Here, we shall not introduce any specific couplings to the SM, but will only estimate the overall signal strength.

The asymmetric DM annihilation rate is suppressed with respect to symmetric DM due to the depleted population of dark antiparticles, albeit this suppression is ameliorated by the larger annihilation cross-section. For asymmetric DM, the expected signal rate is proportional to $Y_{\infty}^{+} Y_{\infty}^{-}\left\langle\sigma_{\text {inel }} v_{\text {rel }}\right\rangle=\left[\eta_{D}^{2} r /(1-r)^{2}\right]\left\langle\sigma_{\text {inel }} v_{\text {rel }}\right\rangle$; for symmetric DM, it is proportional to $\left(Y_{\infty}^{\text {sym }}\right)^{2}\left\langle\sigma_{\text {inel }}^{\text {sym }} v_{\text {rel }}\right\rangle$. Using eq. (2.9) to express $\eta_{D}=\epsilon \eta_{B}$ in terms of $r_{\infty}$, and noting that for symmetric DM, $\Omega_{\mathrm{DM}}=2 Y_{\infty}^{\mathrm{sym}} M_{\mathrm{DM}} \Omega_{\mathrm{B}} /\left(\eta_{B} m_{p}\right)$, we find that the suppression factor of the annihilation signals arising from asymmetric DM with respect to symmetric DM of the same mass is [4]

$$
f_{\mathrm{ID}}=\frac{4 r_{\infty}}{\left(1+r_{\infty}\right)^{2}}\left(\frac{\sigma_{\text {inel }} v_{\text {rel }}}{\sigma_{\text {inel }}^{\text {sym }} v_{\text {rel }}}\right) .
$$

Here, $\sigma_{\text {inel }}$ includes all inelastic processes that contribute to the DM annihilation. Using eq. $(2.18 \mathrm{a})$, and assuming that the ratio $\sigma_{\text {inel }} / \sigma_{\text {inel }}^{\mathrm{sym}}$ is the same during freeze-out and at the velocities relevant for indirect detection, ${ }^{15}$ we obtain the analytical estimate for $f_{\mathrm{ID}}$ in terms of $r_{\infty}$ or $\epsilon$ and $M_{\mathrm{DM}}$,

$$
f_{\mathrm{ID}} \approx \frac{2 r_{\infty} \ln \left(1 / r_{\infty}\right)}{1-r_{\infty}^{2}}=\frac{1}{2}\left[\frac{1-\epsilon^{2} M_{\mathrm{DM}}^{2} /(5 \mathrm{GeV})^{2}}{\epsilon M_{\mathrm{DM}} /(5 \mathrm{GeV})}\right] \ln \left[\frac{1+\epsilon M_{\mathrm{DM}} /(5 \mathrm{GeV})}{1-\epsilon M_{\mathrm{DM}} /(5 \mathrm{GeV})}\right],
$$

where in the second step we used eq. (2.9). For convenience, we define an effective crosssection for estimating the indirect detection signals of asymmetric DM, that can be directly compared to the annihilation cross-section of symmetric DM of the same mass,

$$
\sigma_{\text {ID }} v_{\text {rel }} \equiv \frac{4 r_{\infty}}{\left(1+r_{\infty}\right)^{2}} \sigma_{\text {inel }} v_{\text {rel }} \text {. }
$$

We present the numerical evaluation of $f_{\mathrm{ID}}$ and $\sigma_{\mathrm{ID}} v_{\mathrm{rel}}$ in figs. 6 and 7 . We calculate the cross-sections in the Coulomb regime, using the formulae provided in section 3 . This is a satisfactory approximation in a large range of mediator masses that yield observable signals from the Milky Way and the Dwarfs [24]. We adopt the indicative value $v_{\text {rel }}=10^{-3}$ for the relative velocity of the DM particles, which is a typical value for the Milky Way. ${ }^{16}$ We discern the following regimes:

- For $M_{\mathrm{DM}} \lesssim 10 \mathrm{GeV}$, the DM annihilation occurs mostly in the perturbative regime, both during the DM chemical decoupling in the early universe, and inside haloes today; $\sigma_{\mathrm{ID}} v_{\text {rel }}$ is fairly independent of $M_{\mathrm{DM}}$.

\footnotetext{
${ }^{15}$ This is a good approximation provided that both the freeze-out and the emission of the annihilation signals happen either well within the perturbative regime or well within the Sommerfeld-enhanced regime, for the couplings that correspond both to the symmetric and the asymmetric cases.

${ }^{16}$ Away from the Coulomb regime, i.e. for $v_{\text {rel }} M_{\mathrm{DM}} / 2 \lesssim m_{\text {med }}$, the inelastic cross-sections exhibit resonances, their velocity scaling depends on their partial wave, and BSF has a kinematic cutoff at $m_{\text {med }} \lesssim M_{\mathrm{DM}} \alpha_{D}^{2} / 4[18$, 24]. Moreover, different inelastic processes than those considered in the Coulomb regime may become more dominant [22]. We leave the detailed investigation of these features and their phenomenological implications, in the context of specific asymmetric DM realisations, for future work.
} 


\section{Vector mediator}
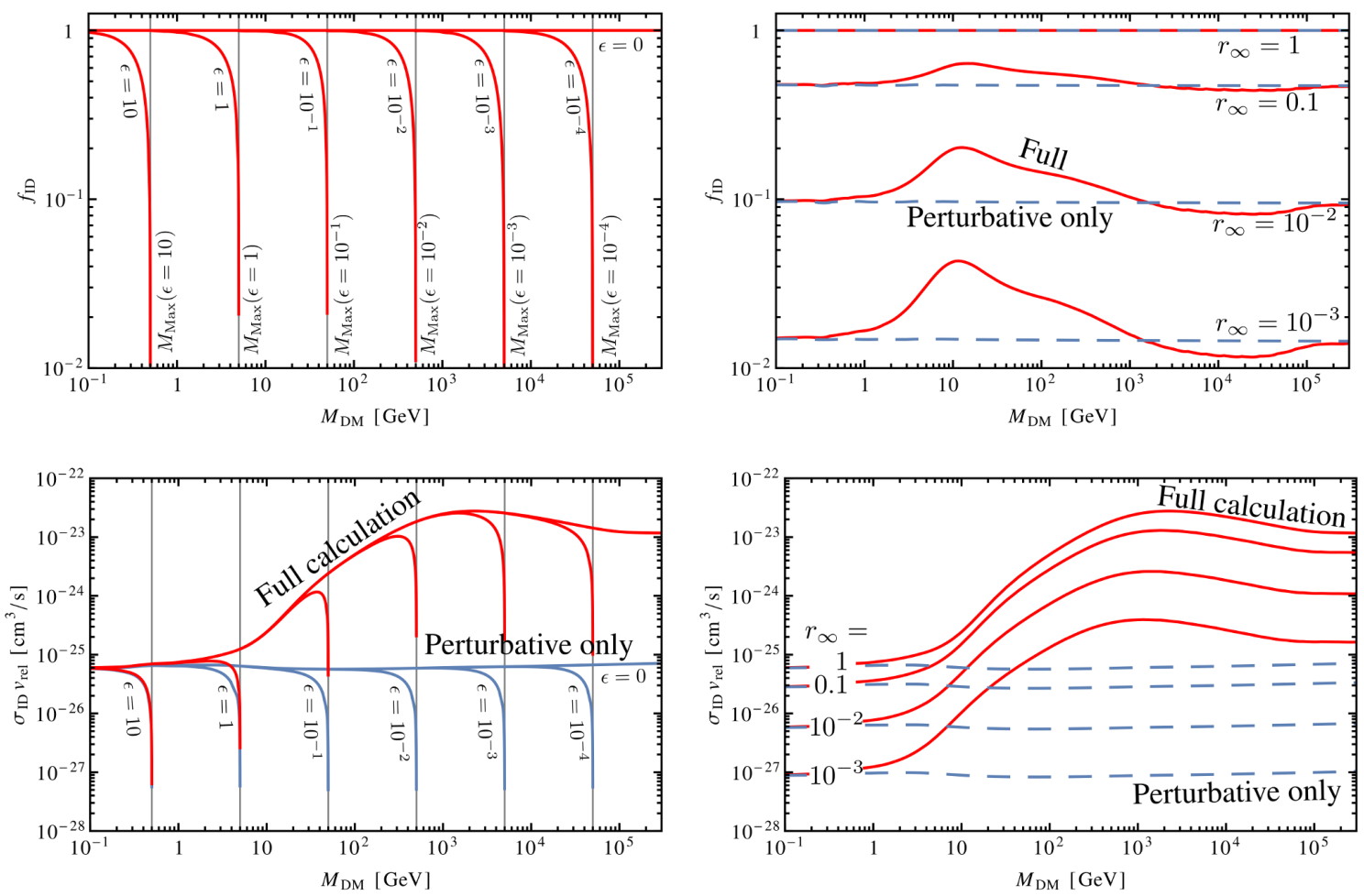

Figure 6. Top: The suppression factor of the expected annihilation signals with respect to symmetric $\mathrm{DM}$ of the same mass, $f_{\mathrm{ID}}$ vs $M_{\mathrm{DM}}$, for fixed $\epsilon=\eta_{D} / \eta_{B}$ (left), and for fixed $r_{\infty}$ (right). Bottom: The effective cross-section for indirect detection signals, $\sigma_{\mathrm{ID}} v_{\text {rel }}=\left[4 r_{\infty} /\left(1+r_{\infty}\right)^{2}\right] \sigma_{\text {inel }} v_{\text {rel }}$ vs $M_{\mathrm{DM}}$, for fixed $\epsilon=\eta_{D} / \eta_{B}$ (left), and for fixed $r_{\infty}$ (right). In all panels, we have used $v_{\text {rel }}=10^{-3}$, which is relevant for indirect searches in the Milky Way, and evaluated the cross-sections in the Coulomb limit, which is a satisfactory approximation within a large range of values of the mediator mass.

- In the intermediate mass range, $M_{\mathrm{DM}} \sim 10 \mathrm{GeV}-1 \mathrm{TeV}$, the Sommerfeld effect has negligible impact during freeze-out, but is significant inside galaxies today, where the average velocity is lower. This essentially sets $\sigma_{0}$ or $\sigma_{1}$ to be independent of $M_{\mathrm{DM}}$, or equivalently $\alpha_{D} \propto M_{\mathrm{DM}}$. Since $S_{\mathrm{ann}}^{(0)}, S_{\mathrm{BSF}} \propto \alpha_{D}$ and $S_{\mathrm{ann}}^{(1)} \propto \alpha_{D}^{3}$ at $\alpha_{D} \gtrsim v_{\text {rel }}$ [cf. eqs. (3.6) and (3.13b)], the effective cross-section for indirect detection signals scales as $\sigma_{\mathrm{ID}} v_{\text {rel }} \propto M_{\mathrm{DM}}$ and $M_{\mathrm{DM}}^{3}$, for fixed $r_{\infty}$, for a vector and a scalar mediator respectively.

- For $M_{\mathrm{DM}} \gtrsim 1 \mathrm{TeV}$, the Sommerfeld effect is operative both during freeze-out and inside haloes today, albeit the enhancement is different due to the different velocity. Consequently, $\sigma_{\mathrm{ID}} v_{\text {rel }}$ becomes again insensitive to $M_{\mathrm{DM}}$, but is significantly larger than what expected from perturbative annihilation, by a factor of $v_{\mathrm{rel}}^{\mathrm{FO}} / v_{\text {rel }}$ and $\left(v_{\text {rel }}^{\mathrm{FO}} / v_{\text {rel }}\right)^{3}$ for a vector and scalar mediator.

It is notable that, due to the Sommerfeld enhancement, $\sigma_{\text {ID }} v_{\text {rel }}$ can be larger, even by many orders of magnitude, than the cross-section for symmetric DM annihilating via contact interactions, for a large range of masses, $M_{\mathrm{DM}} \gtrsim 10 \mathrm{GeV}$, and highly asymmetric DM, in particular $r_{\infty}$ as low as $10^{-3}$ for a vector mediator and $10^{-8}$ for a scalar mediator.

There is, however, an important caveat. Equations (4.1) and (4.2) do not always suf- 


\section{Scalar mediator}
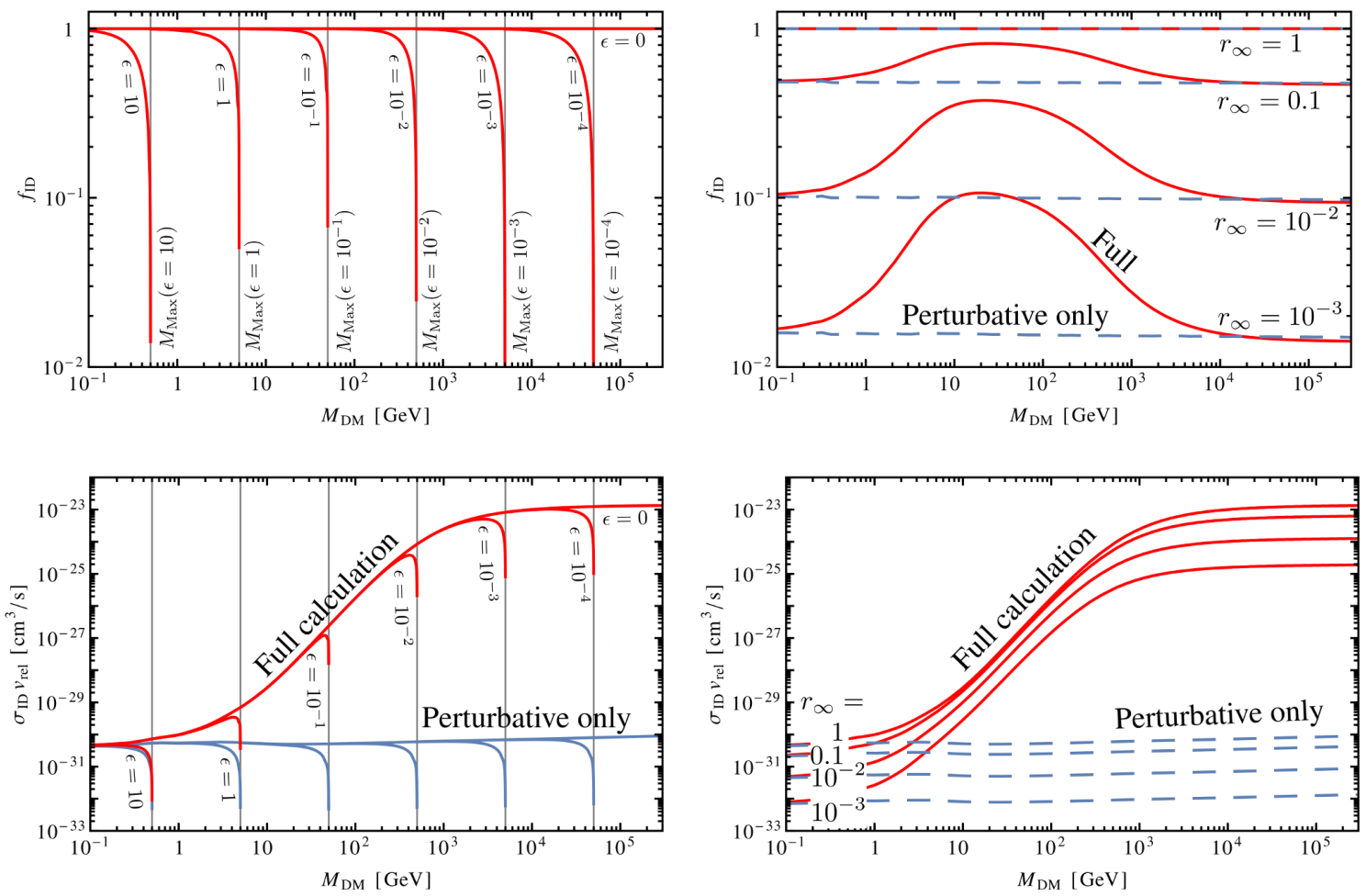

Figure 7. The equivalent of fig. 6, for DM coupled to a light scalar mediator.

fice to predict the indirect detection signals that should be expected from asymmetric DM annihilation. Cosmological events following the DM chemical freeze-out may further suppress the fraction of DM that is available to participate in the annihilation processes at later times. This is, in fact, particularly relevant to scenarios of asymmetric DM coupled to light or massless force mediators.

If DM bares a particle-antiparticle asymmetry and couples to a light or massless dark photon, then gauge invariance mandates that a second dark species, charged under the same dark force, bares an asymmetry and has survived until today, such that the total dark electric charge of the universe vanishes. ${ }^{17}$ While this is self-evident in the case of a massless dark photon implied by an unbroken gauged $U(1)_{D}$, a similar conclusion holds even for a mildly broken $U(1)_{D}$ and a sufficiently light dark photon [42]. The presence of a second asymmetric dark species with the opposite net $U(1)_{D}$ charge also implies that stable atomic bound states can form, thus trapping dark particles that would be otherwise available to participate in annihilation processes with the residual dark antiparticles. The efficiency of dark recombination - the formation of dark atoms - depends on the masses of both species, which demonstrates that eq. (4.2) is insufficient to predict the expected DM annihilation signals. ${ }^{18}$

\footnotetext{
${ }^{17}$ This is, of course, analogous to ordinary protons and electrons in the SM, and to mirror electrons and mirror protons in mirror matter models [36]. For other models of atomic DM, see e.g. [25, 37-41].

${ }^{18}$ As discussed in section 2.1, the additional degrees of freedom in the dark sector will affect $T_{D}$ and hence also the $\left\langle\sigma_{\text {ann }} v_{\text {rel }}\right\rangle$ required to obtain the observed DM abundance. To a good approximation, the required annihilation cross-section scales as $\left\langle\sigma_{\mathrm{ann}} v_{\mathrm{rel}}\right\rangle \propto T_{D}^{\mathrm{FO}} / T_{\mathrm{SM}}^{\mathrm{FO}}$.
} 
Asymmetric DM that couples to a light scalar mediator can form stable bound states due to the attractive nature of the interaction between particles of the same species (as well as between particles and antiparticles). In contrast to the vector mediator case, the formation of particle-particle bound states does not neutralise the interaction mediated by the scalar. This may result in the cosmological formation of a spectrum of multiparticle bound states [43], which of course modifies the number density of the DM states, as well as their annihilation cross-section. In this event, the estimation of the DM annihilation signals based solely on eq. (4.2) would be inaccurate.

The comprehensive computation of the annihilation signals expected in these scenarios merits dedicated analyses that will employ the results presented here, but are beyond the scope of the present work. It is worth noting that in these scenarios, signals for indirect DM searches may also arise from the radiative formation, or the excitation and de-excitation of the stable DM bound states - in particular, from the formation of dark atoms [44] or other related transitions [45] in the case of a vector mediator, and from the formation of dark particle-particle bound states in the case of a scalar mediator [46].

We note in passing that the above cosmological considerations are important also in determining other phenomenological aspects of the scenarios considered here, and in particular the DM self-interactions inside haloes today. The formation of stable bound states typically screens or curtails the DM self-scattering. It is then essential that phenomenological studies take into account the entire cosmological history, of which the DM chemical freeze-out computed here is essentially the first part. 


\section{$5 \quad$ Unitarity limit}

\subsection{Long-range vs. contact-type interactions}

Partial-wave unitarity sets an upper limit on the 2-to-2 total inelastic cross-section. In the non-relativistic regime, it reads [26]

$$
\sigma_{\text {inel }}^{(J)} v_{\text {rel }} \leqslant \sigma_{\text {uni }}^{(J)} v_{\text {rel }}=\frac{4 \pi(2 J+1)}{M_{\mathrm{DM}}^{2} v_{\text {rel }}},
$$

where $J$ denotes the partial wave. Assuming that DM has thermalised in the early universe, the limit (5.1) implies that DM cannot be too heavy, because it would not have annihilated sufficiently, down to its observed density. This consideration was first employed in Ref. [26], to obtain an upper bound on the mass of symmetric thermal-relic DM, which amounts to $\sim 83 \mathrm{TeV}$ for non-self-conjugate DM, using the current measurement of $\Omega_{\mathrm{DM}}$. However, two refined considerations relating to the velocity dependence of $\sigma_{\text {uni }} v_{\text {rel }}$ and the contribution of high partial waves to the inelasticity, raise this upper bound on $M_{\mathrm{DM}}$ considerably. We now expound on these points.

\subsubsection{The velocity dependence of $\sigma_{\text {uni }} v_{\text {rel }}$}

In Ref. [26], the velocity dependence of $\sigma_{\text {uni }} v_{\text {rel }}$ was deemed unphysical, and $v_{\text {rel }}$ in eq. (5.1) was substituted with a constant value of the order of those that occur during freeze-out. More recently, Ref. [15] argued that the velocity dependence of $\sigma_{\text {uni }} v_{\text {rel }}$ has physical significance that needs to be taken into account. It essentially implies that the unitarity limit may be realised only if DM annihilates via a long-range interaction.

For an interaction mediated via a heavy force carrier of mass $m_{\text {med }} \gtrsim M_{\mathrm{DM}}$, the inelastic cross-section scales as $\sigma_{\text {inel }} v_{\text {rel }} \sim \alpha_{D}^{2} M_{\text {DM }}^{2} / m_{\text {med }}^{4}$. Realising the unitarity limit (5.1) via such an interaction would then require a large coupling $\alpha_{D} \sim\left(m_{\text {med }} / M_{\mathrm{DM}}\right)^{2} / \sqrt{v_{\text {rel }}} \gtrsim 1$. Recasting the requirement for realising the unitarity limit, and using the fact that $\alpha_{D} \gtrsim 1$, implies that $m_{\text {med }} \sim \alpha_{D}^{1 / 2} v_{\text {rel }}^{1 / 4} M_{\mathrm{DM}} \lesssim \alpha_{D} M_{\mathrm{DM}}$. This condition states that the range of the interaction between two DM particles, $m_{\mathrm{med}}^{-1}$, is comparable or larger than their Bohr radius, $\left(\alpha_{D} M_{\mathrm{DM}} / 2\right)^{-1}$, and marks precisely the regime where the interaction manifests as long-range, thereby contradicting the original premise of a contact-type interaction. The apparent violation of unitarity indicates that non-perturbative effects associated with the long-range nature of the interaction - namely the Sommerfeld effect, and possibly additional radiative processes, such as BSF - need to be included. (See also Refs. $[9,10]$ for a related discussion.)

Let us also consider the case $m_{\text {med }}<M_{\mathrm{DM}}$. Then, an inelastic cross-section computed perturbatively would scale as $\sigma_{\text {inel }} v_{\text {rel }} \sim \alpha_{D}^{2} / M_{\mathrm{DM}}^{2}$; for example, in a dark QED theory, $\sigma_{\mathrm{ann}} v_{\text {rel }}=\pi \alpha_{D}^{2} / M_{\mathrm{DM}}^{2}$ [cf. eq. (3.5)]. Realising the unitarity limit then requires $\alpha_{D} \sim$ few $/ \sqrt{v_{\text {rel }}} \gtrsim 1>v_{\text {rel }}$. This means that the quantum uncertainty in the momentum exchange between the two interacting particles, which is of the order of the Bohr momentum $\left(M_{\mathrm{DM}} / 2\right) \alpha_{D}$, is larger than the average momentum exchange, $\left(M_{\mathrm{DM}} / 2\right) v_{\text {rel }}$. The 2-particle state is thus not well-approximated by an eigenstate of the relative momentum, and the interaction at infinity needs to be taken into account (see also footnote 14). This amounts to the resummation of the 2-particle-irreducible diagrams that reveals the non-perturbative effects at play.

Including non-perturbative effects results in the inelastic cross-sections exhibiting the same parametric dependence on $M_{\mathrm{DM}}$ and $v_{\text {rel }}$ as eq. (5.1), at least at sufficiently large 
couplings or low velocities (cf. section 3). Therefore, applied to a specific theory with longrange interactions, the unitarity limit simply yields a numerical upper bound on the value of the coupling that merely indicates higher-order terms in any perturbative expansion used in the calculation need to be included.

Therefore, the proper determination of the unitarity constraint on the mass of thermalrelic DM necessitates accounting for the variation of $\sigma_{\text {uni }} v_{\text {rel }}$ with the velocity, in the kinetic equations that describe the DM chemical decoupling in the early universe. This raises the upper bound on the mass of non-self-conjugate symmetric DM, annihilating via $s$-wave processes, to $M_{\text {uni,sym }}^{(J=0)} \approx 140 \mathrm{TeV}$ [15] (see also [47]), assuming that DM annihilates into a thermal bath of the same temperature as the SM. In section 5.2, we extend the computation of this bound to asymmetric DM, using the relic density calculations of the previous section that focused on long-range interactions.

\subsubsection{Higher partial waves}

Higher partial waves may contribute significantly to the total inelastic cross-section, and consequently to the depletion of DM in the early universe.

Reference [26] argued that the $s$-wave contribution dominates the inelastic cross-section, since higher partial waves are suppressed in the non-relativistic regime by $v_{\text {rel }}^{2 J}$. This is indeed true for contact interactions, provided that no symmetry eliminates the $s$-wave contribution. However, the unitarity limit cannot be realised by contact interactions. As discussed above, it may be realised by long-range interactions, which exhibit the same velocity scaling, $\sigma_{\text {inel }}^{(J)} v_{\text {rel }} \propto$ $1 / v_{\text {rel }}$, at large couplings or small $v_{\text {rel }}$, independently of the partial wave [16,33]. Nevertheless, for a process that has a perturbative limit, ${ }^{19}$ the $v_{\text {rel }}^{2 J}$ suppression of the higher partial waves that appears in the perturbative regime $\left(v_{\text {rel }}>\alpha_{D}\right)$, morphs into an $\alpha_{D}^{2 J}$ suppression in the Sommerfeld-enhanced regime $\left(v_{\text {rel }}<\alpha_{D}\right),{ }^{20}$ as already evident in eqs. (3.13). For such processes, it is typically true that the lowest non-vanishing partial wave yields the dominant contribution.

However, the coupling to a light mediator often implies a variety of radiative processes that can annihilate DM. For a vector mediator, the leading order contribution to the direct DM annihilation into radiation is dominantly $s$-wave, while the radiative capture to the ground state is a $p$-wave process, $\mathcal{M}_{\mathrm{BSF}} \propto \sin \theta \propto d_{1,0}^{1}(\theta) .{ }^{21}$ Despite the different partial waves contributing, both the annihilation and BSF have the same dependence on $\alpha_{D}$ for $v_{\text {rel }} \lesssim \alpha_{D}$. Applying the limit (5.1) on eqs. (3.4), using the appropriate value of $J$, we find that unitarity is violated by the leading order computation of $\sigma_{\mathrm{ann}}$ and $\sigma_{\mathrm{BSF}}$ at $\alpha_{D} \gtrsim 0.85$; notably, this is approximately the same value of $\alpha_{D}$ for both processes. Around and above this value of $\alpha_{D}$, higher-order corrections must be included to accurately determine the crosssections of interest. Nevertheless, it is evident that for such large values of $\alpha_{D}$ - around which the unitarity limit on the inelastic cross-sections may be realised - the depletion of $\mathrm{DM}$ via $p$-wave inelastic scattering dominates over $s$-wave, for the velocity range that is relevant to the DM chemical decoupling in the early universe $\left(v_{\text {rel }}^{\mathrm{FO}} \lesssim 0.3\right)$. It is also interesting

\footnotetext{
${ }^{19}$ Not all processes have a perturbative limit, e.g. bound-state formation is inherently non-perturbative and vanishes at small $\alpha_{D} / v_{\text {rel }}$ or small $\alpha_{D} M_{\mathrm{DM}} / m_{\text {med }}$, where $m_{\text {med }}$ is the mediator mass (if non-zero).

${ }^{20}$ This is also true for higher-order corrections in $v_{\text {rel }}^{2}$ within a given partial wave. See Ref. [48] for exact formulae, and footnote 14 for the physical interpretation.

${ }^{21}$ Here, $d_{\lambda_{f}, \lambda_{i}}^{J}(\theta)$ are the Wigner $d$ functions. $\theta$ is the scattering angle, $J$ denotes the partial wave, and $\lambda_{i}=\lambda_{i 1}-\lambda_{i 2}, \lambda_{f}=\lambda_{f 1}-\lambda_{f 2}$ are the initial- and final-state helicities respectively, with the indices 1 and 2 corresponding to the particles of each state. Note that the partial-wave decomposition of $\mathcal{M}_{\mathrm{BSF}}$ was not correctly described in Refs. [15, 16].
} 

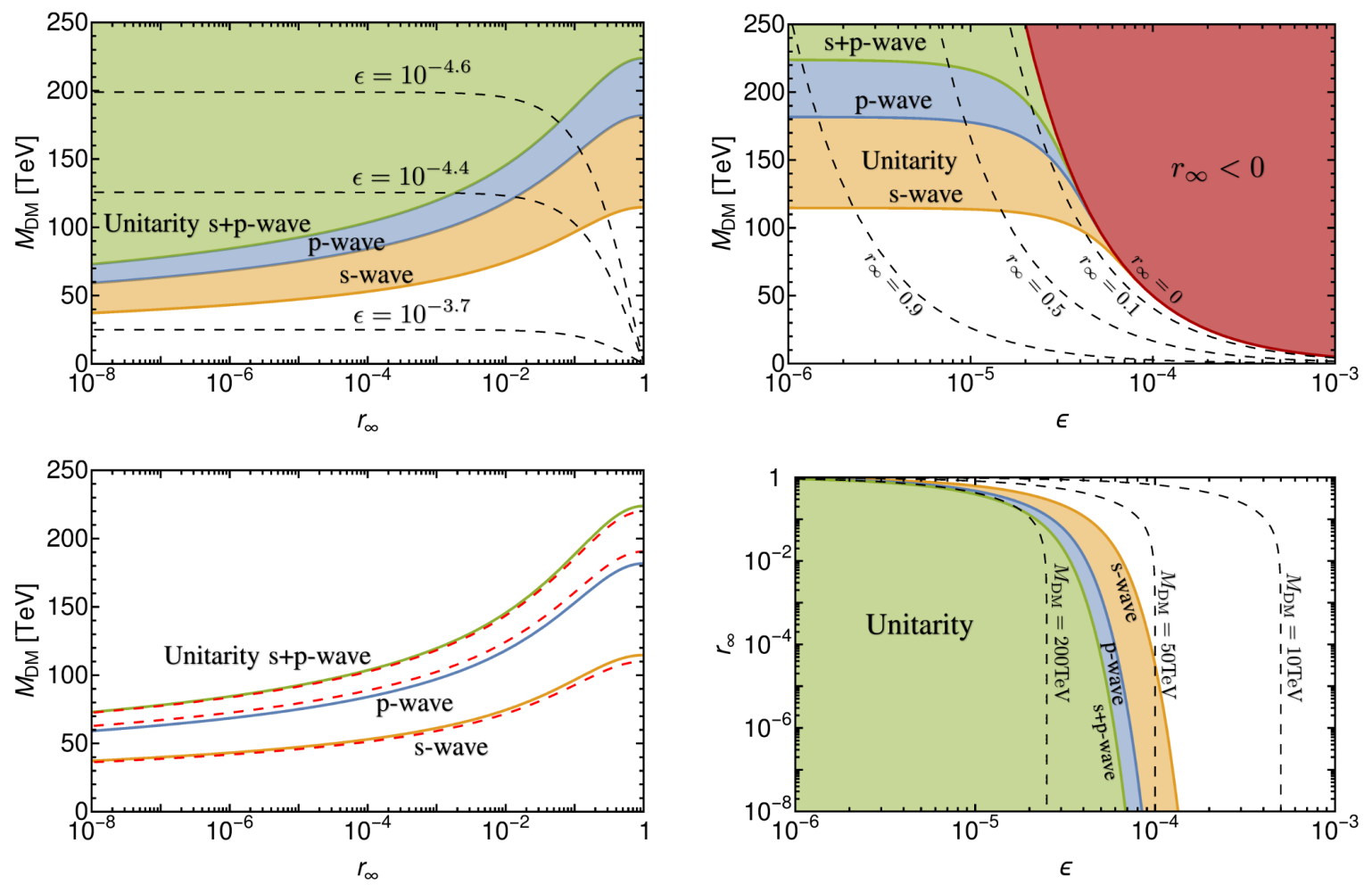

Figure 8. Unitarity bounds on symmetric and asymmetric thermal-relic dark matter, annihilating via processes dominated by the $s$ or $p$ partial waves, or their sum. The $\epsilon, r_{\infty}$ and $M_{\mathrm{DM}}$ dashed contours illustrate the relation $M_{\mathrm{DM}} \simeq(5 \mathrm{GeV} / \epsilon) \times\left(1-r_{\infty}\right) /\left(1+r_{\infty}\right)$ [cf. eq. (2.9)]. Top left: For small values of the final fractional asymmetry $r_{\infty}$, the bounds on $M_{\mathrm{DM}}^{2}$ tighten up approximately logarithmically with decreasing $r_{\infty}$. Top right: At $\epsilon \equiv \eta_{D} / \eta_{B} \ll 10^{-5}$, the DM mass is bounded by the unitarity limit on symmetric thermal-relic DM, while at $\epsilon \gg 10^{-5}$, it is limited by $M_{\max } \simeq 5 \mathrm{GeV} / \epsilon$. Bottom right: For $\epsilon \ll 10^{-5}$, DM retains a large symmetric component, $r_{\infty} \sim \mathcal{O}(1)$, while for $\epsilon \gg 10^{-5}$, small values of $r_{\infty}$ can be attained. Bottom left: Comparison of numerical evaluation (solid) and analytical approximation (dashed) [cf. eqs. (5.4) and (5.6)].

that, around these values of $\alpha_{D}$, the $s$-wave annihilation still gives a sizeable contribution (nearly the maximally allowed by unitarity), and $d$-wave inelastic scattering, which is the dominant mode of the capture into $n=2, \ell=1$ bound states, is comparable to the $s$-wave annihilation [18] (without, though, saturating its unitarity limit). Moreover, for a scalar mediator, the direct annihilation into radiation, which is the dominant inelastic interaction in the Coulomb regime, is a $p$-wave process at leading order, $\mathcal{M}_{\text {ann }} \propto \cos \theta \propto d_{0,0}^{1}(\theta)$. In this case, the apparent violation of unitarity occurs for $\alpha_{D} \gtrsim 1.4$. It is possible that more complex models feature inelastic processes where even higher partial waves dominate (see e.g. Ref. [49] for a model with a rich spectrum of radiative transitions).

From the above it is evident that higher partial waves are important. In section 5.2, we compute the $s$ - and $p$-wave unitarity bounds, and their combination, on the mass of symmetric and asymmetric thermal-relic DM. 


\subsection{Bounds on the mass of symmetric and asymmetric thermal-relic DM}

We first analytically estimate the unitarity bound on the DM mass. The DM relic density depends on the product $\left(1+c / x_{D}^{\mathrm{FO}}\right) \lambda \Phi$, which depends on the annihilation cross-section at freeze-out [cf. eqs. (2.4b) and (2.13a)]. The thermal average of eq. (5.1) is $\left\langle\sigma_{\text {uni }}^{(J)} v_{\text {rel }}\right\rangle=$ $(2 J+1) 4\left(\pi x_{D}\right)^{1 / 2} / M_{\mathrm{DM}}^{2}$. Then,

$$
\begin{aligned}
& \left(1+\frac{c}{x_{D}^{\mathrm{FO}}}\right) \lambda \Phi \leqslant\left(1+\frac{1}{4 x_{D}^{\mathrm{FO}}}\right) \lambda_{\mathrm{uni}} \Phi_{\text {uni }} \\
& =\frac{8 \pi}{3} \sqrt{\frac{g_{*}^{\mathrm{FO}}}{5 x_{D}^{\mathrm{FO}}}}\left(1+\frac{1}{4 x_{D}^{\mathrm{FO}}}\right) \frac{M_{\mathrm{Pl}}}{M_{\mathrm{DM}}} \times \begin{cases}(2 J+1), & \text { solely } J, \\
\left(J_{\max }+1\right)^{2}, & 0 \leqslant J \leqslant J_{\max },\end{cases}
\end{aligned}
$$

depending on which partial waves contribute maximally to the DM annihilation.

For symmetric thermal-relic DM $(\epsilon=0)$, the relic density is [cf. eq. (2.16)]

$$
\Omega_{\mathrm{DM}}=\frac{2 M_{\mathrm{DM}} Y_{\infty}^{\mathrm{sym}} s_{0}}{\rho_{c}} \simeq \frac{2 M_{\mathrm{DM}} s_{0} / \rho_{c}}{\left(1+c / x_{D}^{\mathrm{FO}}\right) \lambda_{\mathrm{sym}} \Phi_{\mathrm{sym}}} \geqslant \frac{2 M_{\mathrm{DM}} s_{0} / \rho_{c}}{\left[1+1 /\left(4 x_{D}^{\mathrm{FO}}\right)\right] \lambda_{\mathrm{uni}} \Phi_{\mathrm{uni}}},
$$

which implies ${ }^{22}$

$$
M_{\mathrm{DM}} \leqslant M_{\text {uni,sym }} \approx 110 \mathrm{TeV} \times \begin{cases}\sqrt{2 J+1}, & \text { solely } J, \\ J_{\max }+1, & 0 \leqslant J \leqslant J_{\max } .\end{cases}
$$

Asymmetric thermal-relic DM requires a larger annihilation cross-section than symmetric DM. Unitarity, thus, sets a tighter upper bound on $M_{\mathrm{DM}}$, that depends on the asymmetry $\epsilon=\eta_{D} / \eta_{B}, M_{\mathrm{DM}} \leqslant M_{\text {uni }}(\epsilon) \leqslant M_{\text {uni,sym. }}$. Moreover, the DM mass is bounded from above by the value it would have if a vanishing late-time fractional asymmetry, $r_{\infty} \rightarrow 0$, could be attained, $M_{\mathrm{DM}}<M_{\max }(\epsilon) \simeq 5 \mathrm{GeV} / \epsilon$ [cf. eq. (2.10)]. $M_{\mathrm{uni}}(\epsilon)$ describes the transition between $M_{\text {uni,sym }}$ and $M_{\max }(\epsilon)$, which occurs at $M_{\max }(\epsilon) \sim M_{\text {uni,sym }}$, i.e. for $\epsilon \sim 5 \mathrm{GeV} / M_{\text {uni,sym }} \sim 10^{-5}$. For $\epsilon \gg 10^{-5}$, the DM mass is bounded essentially by $M_{\max }(\epsilon)$ and very small $r_{\infty}$ can be realised; for $\epsilon \ll 10^{-5}, M_{\mathrm{DM}}$ is bounded by $M_{\mathrm{uni} \text {,sym }}$ and $r_{\infty} \sim \mathcal{O}(1)$. Conversely, the unitarity limit may be interpreted as a lower bound on the fractional asymmetry, $r_{\infty} \geqslant r_{\infty, \text { uni }}$; for small $\epsilon$ or large $M_{\mathrm{DM}}, r_{\infty}$ cannot be too small, which in turn may result in significant annihilation signals at late times.

We may now estimate $M_{\text {uni }}$ in the presence of an asymmetry. The expansion of eq. (2.18) gives

$$
\ln r_{\infty} \simeq-\left(1+\frac{c}{x_{D}^{\mathrm{FO}}}\right) \eta_{D} \lambda \Phi \gtrsim-\left(1+\frac{1}{4 x_{D}^{\mathrm{FO}}}\right) \epsilon \eta_{B} \lambda_{\text {uni }} \Phi_{\text {uni }}
$$

${ }^{22}$ In terms of the various parameters involved,

$$
M_{\mathrm{uni}, \mathrm{sym}}^{(J=0)}=\left[\frac{4 \pi}{3} \sqrt{\frac{g_{*}^{\mathrm{FO}}}{5 x_{D}^{\mathrm{FO}}}}\left(1+\frac{1}{4 x_{D}^{\mathrm{FO}}}\right) \frac{\rho_{c} M_{\mathrm{P} 1} \Omega_{\mathrm{DM}}}{s_{0}}\right]^{1 / 2} \approx 110 \mathrm{TeV}
$$

Note that there is some sensitivity on the assumptions about $\tilde{T}$ and the degrees of freedom in each sector, via $g_{*}^{\mathrm{FO}}$. Had we assumed that the dark plasma is at the same temperature as the SM plasma at the time of freezeout, we would have found $M_{\mathrm{uni}, \mathrm{sym}}^{(J=0)} \approx 140 \mathrm{TeV}$. According to the assumptions made here, for $M_{\mathrm{DM}} \sim 100 \mathrm{TeV}$, the dark plasma is at a somewhat higher temperature than the SM at the time of DM freeze-out. This is due to the DM degrees of freedom becoming non-relativistic below the last common temperature $\tilde{T}$ of the two sectors, while no decoupling of SM degrees of freedom has yet occurred at $T_{\mathrm{SM}} \sim 100 \mathrm{TeV} / x_{D}^{\mathrm{FO}} \sim$ few TeV. A hotter dark sector necessitates a larger DM annihilation cross-section, and therefore implies a stronger unitarity bound on $M_{\mathrm{DM}}$. 
where $\lambda_{\text {uni }} \Phi_{\text {uni }}$ is given in eq. (5.2), and $\epsilon, r_{\infty}$ and $M_{\mathrm{DM}}$ are also related via eq. (2.9). Solving eq. (2.9) for $\epsilon, r_{\infty}$ and $M_{\mathrm{DM}}$, and substituting into eq. (5.5), we obtain respectively

$$
\begin{gathered}
M_{\text {uni }} \approx M_{\text {uni,sym }} \times\left[\left(\frac{1+r_{\infty}}{1-r_{\infty}}\right) \frac{\ln \left(1 / r_{\infty}\right)}{2}\right]^{-1 / 2}, \\
M_{\text {uni }} \times \sqrt{\frac{1}{2}\left(\frac{5 \mathrm{GeV}}{\epsilon M_{\mathrm{uni}}}\right) \ln \left[\frac{1+\epsilon M_{\mathrm{uni}} /(5 \mathrm{GeV})}{1-\epsilon M_{\mathrm{uni}} /(5 \mathrm{GeV})}\right]} \approx M_{\mathrm{uni}, \mathrm{sym}}, \\
r_{\infty, \mathrm{uni}}=\exp \left[-2\left(\frac{1+r_{\infty, \mathrm{uni}}}{1-r_{\infty, \mathrm{uni}}}\right)\left(\frac{\epsilon}{5 \mathrm{GeV} / M_{\mathrm{uni}, \mathrm{sym}}}\right)^{2}\right],
\end{gathered}
$$

where $M_{\text {uni,sym }}$ is given in eq. (5.4). The last two equations can be solved numerically to obtain $M_{\text {uni }}$ and $r_{\infty, \text { uni }}$ in terms of $\epsilon$.

Equations (5.4) and (5.6) provide an analytical approximation to the bounds implied by unitarity, on symmetric and asymmetric thermal-relic DM. In fig. 8, we present the numerical computation of these bounds, for DM annihilation dominated by the $s$ and $p$ partial waves, or their sum.

\section{Conclusion}

In a variety of models, asymmetric DM is hypothesised to couple to light force mediators. Models with hidden sectors are motivated either by high-energy physics, such as stringtheory constructions (see e.g. [50]), and/or on phenomenological grounds, for example by the similarity of the DM and ordinary matter densities (e.g. [38, 39, 41, 50]), self-interacting DM (e.g. $[42,51,52])$ and dissipative DM $[25,36,40,53-55]$. This category also encompasses asymmetric WIMP DM with TeV-scale mass; indeed, for multi-TeV particles, the Weak interactions of the SM, mediated by $\sim 100 \mathrm{GeV}$ gauge bosons, manifest as long-range $[9,10]$. In the present work, we focused on minimal models that feature long-range dynamics. We computed the DM freeze-out in the early universe, estimated the resulting annihilation signals at late times, and deduced constraints implied by unitarity.

Due to the Sommerfeld enhancement of the inelastic processes, the couplings required to eliminate efficiently the dark antiparticles and establish a large final asymmetry $\left(r_{\infty} \ll 1\right)$, can be considerably lower than in the case of contact interactions. Within a specific model, this broadens the low-energy parameter space that yields highly asymmetric DM.

Despite lowering the predicted couplings, the Sommerfeld effect implies that the indirect detection signals from the annihilations of the residual dark antiparticles can be significant. For example, for $M_{\mathrm{DM}} \gtrsim \mathrm{TeV}$ and final fractional asymmetry as low as $r_{\infty} \sim 10^{-3}$, the annihilation rate of asymmetric DM coupled to a light dark photon may be larger than that expected from symmetric DM with contact interactions. Figures 6 and 7 illustrate this point. This opens up the possibility of probing asymmetric DM with observations of the CMB, the Milky Way and the Dwarf galaxies. Moreover, the capture of DM in the interior of the Sun and its annihilation via metastable mediators can give rise to enhanced neutrino signals due to reduced absorption [56], and offers another opportune probe [7, 57-59]. However, we emphasise that the accurate determination of the expected annihilation signals necessitates that the entire cosmological history of any model of interest is first carefully considered; this may involve events such as the cosmological formation of stable bound states [42, 43], that would suppress the annihilation signals estimated here. In fact, the radiative formation of 
stable bound states inside haloes is itself a potential source of indirect signals, albeit of lower energy [44, 46].

Long-range interactions, if adequately strong, can maximise the probability for inelastic scattering. In this regime of large inelasticity, higher partial waves can yield a significant, and in some cases the dominant contribution. However, even maximal inelasticity suffices to annihilate a thermal particle density down to the observed DM abundance only if these particles are not too heavy. The upper bound on the mass of thermal-relic DM implied by unitarity strengthens as the DM asymmetry increases. Conversely, unitarity implies that very heavy $\mathrm{DM}$, or $\mathrm{DM}$ with small particle-minus-antiparticle-number-to-entropy ratio $\eta_{D}$, has a significant symmetric component today. The unitarity bounds are shown in fig. 8 .

\section{Acknowledgments}

I.B. thanks Kai Schmidt-Hoberg for comments on the manuscript. K.P. thanks Adam Falkowski and Andreas Goudelis for very helpful discussions on unitarity. K.P. was supported by the ANR ACHN 2015 grant ("TheIntricateDark" project), and by the NWO Vidi grant "Self-interacting asymmetric dark matter".

\section{References}

[1] K. Petraki and R. R. Volkas, Review of asymmetric dark matter, Int.J.Mod.Phys. A28 (2013) $1330028,[1305.4939]$.

[2] R. J. Scherrer and M. S. Turner, On the Relic, Cosmic Abundance of Stable Weakly Interacting Massive Particles, Phys. Rev. D33 (1986) 1585.

[3] K. Griest and D. Seckel, Cosmic Asymmetry, Neutrinos and the Sun, Nucl.Phys. B283 (1987) 681.

[4] M. L. Graesser, I. M. Shoemaker and L. Vecchi, Asymmetric WIMP dark matter, JHEP 1110 (2011) 110, [1103.2771].

[5] H. Iminniyaz, M. Drees and X. Chen, Relic Abundance of Asymmetric Dark Matter, JCAP 1107 (2011) 003, [1104.5548].

[6] N. F. Bell, S. Horiuchi and I. M. Shoemaker, Annihilating Asymmetric Dark Matter, Phys.Rev. D91 (2015) 023505, [1408.5142].

[7] K. Murase and I. M. Shoemaker, Detecting Asymmetric Dark Matter in the Sun with Neutrinos, Phys. Rev. D94 (2016) 063512, [1606.03087].

[8] A. Sommerfeld, Über die Beugung und Bremsung der Elektronen, Ann. Phys. 403 (1931) 257-330.

[9] J. Hisano, S. Matsumoto and M. M. Nojiri, Unitarity and higher order corrections in neutralino dark matter annihilation into two photons, Phys.Rev. D67 (2003) 075014, [hep-ph/0212022].

[10] J. Hisano, S. Matsumoto and M. M. Nojiri, Explosive dark matter annihilation, Phys.Rev.Lett. 92 (2004) 031303, [hep-ph/0307216].

[11] M. Pospelov and A. Ritz, Astrophysical Signatures of Secluded Dark Matter, Phys.Lett. B671 (2009) 391-397, [0810.1502].

[12] J. D. March-Russell and S. M. West, WIMPonium and Boost Factors for Indirect Dark Matter Detection, Phys.Lett. B676 (2009) 133-139, [0812.0559].

[13] W. Shepherd, T. M. Tait and G. Zaharijas, Bound states of weakly interacting dark matter, Phys.Rev. D79 (2009) 055022, [0901.2125]. 
[14] J. L. Feng, M. Kaplinghat, H. Tu and H.-B. Yu, Hidden Charged Dark Matter, JCAP 0907 (2009) 004, [0905.3039].

[15] B. von Harling and K. Petraki, Bound-state formation for thermal relic dark matter and unitarity, JCAP 12 (2014) 033, [1407.7874].

[16] K. Petraki, M. Postma and M. Wiechers, Dark-matter bound states from Feynman diagrams, JHEP 1506 (2015) 128, [1505.00109].

[17] S. P. Liew and F. Luo, Effects of QCD bound states on dark matter relic abundance, JHEP 02 (2017) 091, [1611.08133].

[18] K. Petraki, M. Postma and J. de Vries, Radiative bound-state-formation cross-sections for dark matter interacting via a Yukawa potential, 1611.01394.

[19] J. Hisano, S. Matsumoto, M. M. Nojiri and O. Saito, Non-perturbative effect on dark matter annihilation and gamma ray signature from galactic center, Phys.Rev. D71 (2005) 063528, [hep-ph/0412403].

[20] N. Arkani-Hamed, D. P. Finkbeiner, T. R. Slatyer and N. Weiner, A Theory of Dark Matter, Phys.Rev. D79 (2009) 015014, [0810.0713].

[21] H. An, M. B. Wise and Y. Zhang, Effects of Bound States on Dark Matter Annihilation, 1604.01776.

[22] H. An, M. B. Wise and Y. Zhang, Strong CMB Constraint On P-Wave Annihilating Dark Matter, 1606.02305.

[23] T. Bringmann, F. Kahlhoefer, K. Schmidt-Hoberg and P. Walia, Strong constraints on self-interacting dark matter with light mediators, 1612.00845.

[24] M. Cirelli, P. Panci, K. Petraki, F. Sala and M. Taoso, Dark Matter's secret liaisons: phenomenology of a dark U(1) sector with bound states, 1612.07295.

[25] P. Agrawal, F.-Y. Cyr-Racine, L. Randall and J. Scholtz, Dark Catalysis, 1702.05482.

[26] K. Griest and M. Kamionkowski, Unitarity Limits on the Mass and Radius of Dark Matter Particles, Phys.Rev.Lett. 64 (1990) 615.

[27] A. Kusenko, Small Q balls, Phys.Lett. B404 (1997) 285, [hep-th/9704073].

[28] A. Kusenko, Solitons in the supersymmetric extensions of the standard model, Phys.Lett. B405 (1997) 108, [hep-ph/9704273].

[29] A. Kusenko and M. E. Shaposhnikov, Supersymmetric $Q$ balls as dark matter, Phys.Lett. B418 (1998) 46-54, [hep-ph/9709492].

[30] G. Belanger, F. Boudjema, A. Pukhov and A. Semenov, MicrOMEGAs: A Program for calculating the relic density in the MSSM, Comput. Phys. Commun. 149 (2002) 103-120, [hep-ph/0112278].

[31] Planck collaboration, P. A. R. Ade et al., Planck 2015 results. XIII. Cosmological parameters, Astron. Astrophys. 594 (2016) A13, [1502.01589].

[32] P. Adshead, Y. Cui and J. Shelton, Chilly Dark Sectors and Asymmetric Reheating, JHEP 06 (2016) 016, [1604.02458].

[33] S. Cassel, Sommerfeld factor for arbitrary partial wave processes, J.Phys. G37 (2010) 105009, [0903.5307].

[34] E. W. Kolb and M. S. Turner, The Early universe, Front.Phys. 69 (1990) 1-547.

[35] T. Lin, H.-B. Yu and K. M. Zurek, On Symmetric and Asymmetric Light Dark Matter, Phys.Rev. D85 (2012) 063503, [1111.0293].

[36] R. Foot, Mirror dark matter: Cosmology, galaxy structure and direct detection, Int.J.Mod.Phys. 
A29 (2014) 1430013, [1401.3965].

[37] D. E. Kaplan, G. Z. Krnjaic, K. R. Rehermann and C. M. Wells, Atomic Dark Matter, JCAP 1005 (2010) 021, [0909.0753].

[38] K. Petraki, M. Trodden and R. R. Volkas, Visible and dark matter from a first-order phase transition in a baryon-symmetric universe, JCAP 1202 (2012) 044, [1111.4786].

[39] B. von Harling, K. Petraki and R. R. Volkas, Affleck-Dine dynamics and the dark sector of pangenesis, JCAP 1205 (2012) 021, [1201.2200].

[40] R. Foot and S. Vagnozzi, Dissipative hidden sector dark matter, Phys.Rev. D91 (2015) 023512, [1409.7174].

[41] J. Choquette and J. M. Cline, Minimal non-Abelian model of atomic dark matter, Phys. Rev. D92 (2015) 115011, [1509.05764].

[42] K. Petraki, L. Pearce and A. Kusenko, Self-interacting asymmetric dark matter coupled to a light massive dark photon, JCAP 1407 (2014) 039, [1403.1077].

[43] M. B. Wise and Y. Zhang, Stable Bound States of Asymmetric Dark Matter, Phys.Rev. D90 (2014) 055030, [1407.4121].

[44] L. Pearce, K. Petraki and A. Kusenko, Signals from dark atom formation in halos, Phys.Rev. D91 (2015) 083532, [1502.01755].

[45] J. M. Cline, Y. Farzan, Z. Liu, G. D. Moore and W. Xue, 3.5keV x rays as the 21cm line of dark atoms, and a link to light sterile neutrinos, Phys.Rev. D89 (2014) 121302, [1404.3729].

[46] L. Pearce and A. Kusenko, Indirect Detection of Self-Interacting Asymmetric Dark Matter, Phys.Rev. D87 (2013) 123531, [1303.7294].

[47] C. F. Berger, L. Covi, S. Kraml and F. Palorini, The Number density of a charged relic, JCAP 0810 (2008) 005, [0807.0211].

[48] S. El Hedri, A. Kaminska and M. de Vries, A Sommerfeld Toolbox for Colored Dark Sectors, 1612.02825.

[49] P. Asadi, M. Baumgart, P. J. Fitzpatrick, E. Krupczak and T. R. Slatyer, Capture and Decay of Electroweak WIMPonium, 1610.07617.

[50] J. J. Heckman and S.-J. Rey, Baryon and Dark Matter Genesis from Strongly Coupled Strings, JHEP 1106 (2011) 120, [1102.5346].

[51] F.-Y. Cyr-Racine and K. Sigurdson, The Cosmology of Atomic Dark Matter, Phys.Rev. D87 (2013) 103515, [1209.5752].

[52] J. M. Cline, Z. Liu, G. Moore and W. Xue, Scattering properties of dark atoms and molecules, Phys.Rev. D89 (2014) 043514, [1311.6468].

[53] R. Foot, Dissipative dark matter and the rotation curves of dwarf galaxies, JCAP 1607 (2016) 011, [1506.01451].

[54] J. Fan, A. Katz, L. Randall and M. Reece, Double-Disk Dark Matter, Phys.Dark Univ. 2 (2013) 139-156, [1303.1521].

[55] K. K. Boddy, M. Kaplinghat, A. Kwa and A. H. G. Peter, Hidden Sector Hydrogen as Dark Matter: Small-scale Structure Formation Predictions and the Importance of Hyperfine Interactions, Phys. Rev. D94 (2016) 123017, [1609.03592].

[56] N. F. Bell and K. Petraki, Enhanced neutrino signals from dark matter annihilation in the Sun via metastable mediators, JCAP 1104 (2011) 003, [1102.2958].

[57] ANTARES collaboration, M. Ardid and C. Tnnis, Constraining Secluded Dark Matter models with the ANTARES neutrino telescope, PoS ICRC2015 (2016) 1212. 
[58] ANTARES collaboration, S. Adrin-Martnez et al., A search for Secluded Dark Matter in the Sun with the ANTARES neutrino telescope, JCAP 1605 (2016) 016, [1602.07000].

[59] M. Ardid, I. Felis, A. Herrero and J. A. Martnez-Mora, Constraining Secluded Dark Matter models with the public data from the 79-string IceCube search for dark matter in the Sun, 1701.08863. 